\title{
Examining the role of traditional masculinity and depression in men's risk for contracting COVID-19
}

Walther, A. ${ }^{1, *}$, Eggenberger, L. ${ }^{1}$, Grub, J. ${ }^{1}$, Ogrodniczuk, J.S. ${ }^{2}$, Seidler, Z.E. ${ }^{3,4}$, Rice, S.M. ${ }^{3,4}$, Kealy, D. ${ }^{2}$, Oliffe, J.L. ${ }^{5,6}$, Ehlert, U. ${ }^{1}$

${ }^{1}$ Clinical Psychology and Psychotherapy, University of Zurich, Zurich, Switzerland

${ }^{2}$ Department of Psychiatry, University of British Columbia, Vancouver, Canada

${ }^{3}$ Centre for Youth Mental Health, The University of Melbourne, Parkville, VIC, Australia

${ }^{4}$ Orygen, Melbourne, VIC, Australia

${ }^{5}$ School of Nursing, University of British Columbia, Vancouver, Canada

${ }^{6}$ Department of Nursing, University of Melbourne, Victoria, Australia

${ }^{*}$ Corresponding author

Running head: Traditional masculinity and the risk of contracting COVID-19

Words (Text): 5’983, Words (Abstract): 273, References: 58, Tables: 2, Figures: 4,

Supplementary Figures: 1, Supplementary-Tables: 3

Correspondence to: Dr. Andreas Walther, Binzmühlestrasse 14, 8050, Zürich; Clinical Psychology and Psychotherapy, University of Zurich, Zurich, Switzerland

a.walther@psychologie.uzh.ch

Submitted to Preprint Server: PsyArXiv 


\begin{abstract}
Background: In the light of the COVID-19 pandemic and claims that traditional masculinity may put some men at increased risk for infection, research reporting men's health behaviors is critically important. Traditional masculine norms such as self-reliance and toughness are associated with a lower likelihood to vaccinate or follow safety restrictions. Furthermore, infection risk and traditional masculinity should be investigated in a differentiated manner including gender role orientation, underlying traditional masculine ideologies and male gender role conflict.
\end{abstract}

Methods: In this pre-registered online survey conducted during March/April 2021 in German-speaking countries in Europe, 490 men completed questionnaires regarding contracting COVID-19 as confirmed by a validated test, fear of COVID-19 (FCV-19S), and experience of psychological burden due to COVID-19. In addition, depression symptomatology was assessed by using prototypical internalizing and male-typical externalizing depression symptoms. Furthermore, self-identified masculine gender orientation, endorsement of traditional masculine ideologies, and gender role conflict were measured.

Results: A total of $6.9 \%$ of men $(n=34)$ reported having contracted COVID-19 since the beginning of the pandemic. Group comparisons revealed that men who had contracted COVID-19 exhibited higher overall traditional masculine ideology and gender role conflict. Logistic regression controlling for confounders (age, income, education, and sexual orientation) indicated that only depression symptoms are independently associated with the risk of having contracted COVID-19. While prototypical depression symptoms were negatively associated with the risk of having contracted COVID-19, male-typical externalizing depression symptoms were positively associated with the risk of having contracted COVID-19. 
Conclusion: For traditional masculinity, no robust association for an increased risk of contracting COVID-19 could be established, while higher male-typical externalizing depression symptoms were associated with an increased risk of having contracted COVID-19. Key words: Traditional masculinity; traditional male role norms; COVID-19; depression; MDRS-22

Public significance statement: Understanding the factors that contribute to increased risk of contracting COVID-19 is essential to developing tailored prevention strategies. Traditional masculinity appears only modestly related with an increased risk of contracting COVID-19, while for elevated levels of male-typical externalizing depression symptoms rooted in traditional masculine ideologies, robust associations emerged. Men in the present sample with high externalizing depression symptoms appear to be more likely to contract COVID-19. 


\section{Introduction}

The COVID-19 pandemic poses major health and economic ramifications worldwide and effectively responding to the pandemic remains an ongoing challenge. Investigation of the pandemic is critical to ending it, and better controlling future pandemics (Li et al., 2021). As one such central concept that might underlie the increased spread of the COVID-19 virus, traditional masculinity is increasingly coming into focus (Bridges et al., 2021).

Although men and women were infected with COVID-19 to a similar extent at the beginning of the pandemic, men were much more likely to die from COVID-19 (Peckham et al., 2020). Research indicates that the cause of the increased mortality rate in infected men was not rooted in male biology but in men's health risk behaviors such as smoking and drinking, which can complicate COVID-19 infections and may lead to a more severe disease course (Hamer et al., 2020; Peckham et al., 2020). Traditional masculinity in connection with health risk behavior has become increasingly important in explaining why specific subpopulations adhere less to state-imposed protective measures such as mask wearing or show increased mortality (Bridges et al., 2021).

Traditional masculinity has long been related to men's health risk behaviors (Courtenay, 2000; Harrison, 1978). This is reflected by consistent findings of gender differences emerging from the current pandemic showing that men were found to be more likely than women to downplay the risks associated with COVID-19 (Umamaheswar \& Tan, 2020). Men were also less likely than women to report fearing "very serious" consequences if they became infected (Babalola et al., 2020; Capraro \& Barcelo, 2020). Men in general were also less likely to follow public health protocols (Clark et al., 2020; Haischer et al., 2020), including refusing to wear masks, demonstrating greater negative affect towards wearing a mask, and less reported handwashing and social distancing compared to women (Capraro \& Barcelo, 2020; Haischer et al., 2020; Okten et al., 2020). Relative to women, men are more likely to believe that wearing a face mask is embarrassing or a sign of weakness (Capraro \& 
Barcelo, 2020) and Palmer and Peterson (2020) specifically linked stronger endorsement of the traditional male role norm of toughness with greater levels of negativity towards mask wearing. Taken together, men in general show poorer adherence to protection measures and health behaviors and therefore are at increased risk for contracting COVID-19 as compared to women (Capraro \& Barcelo, 2020; Cassino \& Besen-Cassino, 2020; Mahalik et al., 2021; Palmer \& Peterson, 2020).

These hesitant attitudes and behaviors to follow state-imposed protection measures for ending the COVID-19 pandemic by many men appear to be based on socially constructed and idealized male gender norms of strength, toughness, and self-reliance adopted during men's gender role socialization (Levant \& Richmond, 2015; O’Neil, 2013). Thus, studies comparing men with low and high adherence or conformity to traditional male role norms should provide further insight into the relation between traditional masculinity, following protection measures and the risk for contracting COVID-19. However, to date there are few available studies on this topic. A non-peer reviewed but publicly available report highlighted those men who identified as "completely masculine" were nearly three times more likely to report having been diagnosed with COVID-19 than men self-identifying as "mostly masculine" or "slightly masculine" (Dan Cassino, 2021). Furthermore, the same authors reported that men selfidentifying as "completely masculine" expressed less intention to get vaccinated for COVID19 as men self-identifying as "mostly masculine" or "slightly masculine"(Cassino \& BesenCassino, 2020). Mahalik and colleagues provided preliminary evidence that higher conformity to traditional masculine norms is associated with negative attitudes toward mask wearing during the ongoing pandemic and that conformity to traditional masculine norms interacts with more conservative political ideologies for the prediction of mask wearing attitudes (Mahalik et al., 2021).

The relationship between traditional masculine role norms and health behaviors or health outcomes in general is, however, complex. Greater adherence to traditional masculinity 
was initially associated with worse health behaviors or increased mortality (Lippa et al., 2000). A meta-analytic investigation revealed that conformity to traditional masculine norms is associated with overall worse mental health outcomes and reduced help seeking (Wong et al., 2017). Moreover, studies indicate traditional masculinity to be associated with delayed therapy initiation (Eggenberger et al., 2021), and increased risk for suicide (Coleman et al., 2020; Walther, Grub, Tsar, et al., 2021). However, more recently also positive and health promoting aspects of traditional masculinity are being recognized (Gerdes \& Levant, 2018). The conceptualization and operationalization of masculinity has evolved over time with its beginning in the gender role identity paradigm attributing gender-typical traits to men and women (Bem, 1974; Pleck, 1981) being followed by the gender role strain paradigm focusing on the adherence and conformity to traditional masculine role norms (Levant et al., 1992; Mahalik et al., 2003; O'Neil et al., 1986). Based on the social constructionist understanding of masculinity Levant and Wimer (2014), for example, revealed that conforming to certain masculine norms act as protective buffers for some health behaviors, while others are consistently identified as risk factors. This was corroborated in a subsequent study showing that the traditional masculine role norm of primacy of work was mostly related with positive health outcomes, while for four other role norms (winning, risk-taking, pursuit of status, and disdain for homosexuals) a balance of positive and negative health outcomes was observed (Gerdes \& Levant, 2018). Yet, six subscales (emotional control, violence, power over women, dominance, playboy, and self-reliance) were predominantly related to negative health outcomes (Gerdes \& Levant, 2018). Therefore, besides reliance on total scores of commonly used masculinity measures, which often obscure the complexity of associations, subscales highlighting particular male role norms should be investigated and reported.

In addition, it is increasingly being discussed whether individuals with mental disorders might be at increased risk for contracting COVID-19 due to potential cognitive deficits including executive dysfunction, negative health behaviors (e.g. smoking), or 
structural barriers hindering the ability to successfully quarantine at home (Shinn \& Viron, 2020; Yao et al., 2020). While there are reports suggesting depression is associated with an increased risk for contracting COVID-19 (Chang et al., 2020; Tang et al., 2020; Taquet et al., 2021; Wang et al., 2021), a similar number of studies found no association or a reduced risk for contracting COVID-19 in these individuals (Izurieta et al., 2021; Jeon et al., 2021; Nemani et al., 2021; Salvatore et al., 2021). There is also evidence that some individuals intentionally expose themselves to the virus with the intent of self-harm or to suicide, further suggesting increased risk for contracting COVID-19 among individuals with mental health problems (Ammerman et al., 2021). However, a meta-analysis examining the relation between mood disorders and risk of contracting COVID-19 in over 91 million individuals could not identify significant associations, suggesting the need to examine more specific subgroups regarding this question (Ceban et al., 2021).

As no association was identified in the above-mentioned meta-analysis on the relation between mood disorders and the risk of contracting COVID-19 (Ceban et al., 2021), it emerges as relevant to investigate a more fine-grained research question regarding how certain disorders, and indeed their unique symptomatology, are associated with a risk of contracting COVID-19. Considering that individuals with prototypical depression symptoms such as depressive mood, anhedonia, or fatigue show increased social withdrawal, higher rates of social isolation and unemployment, one would assume a reduced risk of contracting COVID-19 due to the reduced social interactions (Ceban et al., 2021). By contrast, men with high traditional masculinity exhibit more male-typical externalizing depression symptomatology (Eggenberger et al., 2021; Rice et al., 2013; Walther \& Seidler, 2020), which is characterized by anger and aggression, risk taking, or substance misuse (Cavanagh et al., 2017; Martin et al., 2013; Rice et al., 2013; Walther, Grub, Ehlert, et al., 2021; Winkler et al., 2005). Such a symptom pattern might be assumed to be associated with an increased risk of contracting COVID-19. Risk-taking, for example, is characterized by not caring about the 
consequences of one's actions, so men experiencing externalizing depression symptomatology may care less about their own well-being as well as the well-being of those around them.

The aim of the present study was to investigate the relationship between traditional masculinity measured by different conceptual constructs (gender role orientation, traditional masculine ideology, gender role conflict), depressive symptomatology (prototypical and maletypical externalizing depression symptoms) and self-reported infection with COVID-19. Based on the outlined literature, we hypothesize traditional masculinity to be positively associated with having contracted COVID-19. We hypothesize this relationship uniformly for all masculinity constructs and their respective subscales. The a priori formulated directed hypothesis are retrievable under OSF (https://osf.io/q4pw3/ [embargo till 2021/12/31]). To better understand the relation between specific depression symptomatology and risk of contracting COVID-19, we further investigated in an explorative manner whether men with higher prototypical depression symptoms are less likely to have contracted COVID-19 and whether men with higher male-typical externalizing depression symptoms are more likely to have contracted COVID-19.

\section{Methods}

\subsection{Sample and Procedure}

This anonymous, cross-sectional online survey entitled 'Men's Mental Health in Times of COVID-19' was pre-registered and approved by the local ethics committee of the Faculty of Arts and Social Sciences of the University of Zurich (Authorization No. 21.2.4). Following the Open Science standards, a priori defined study hypotheses, statistical analyses, and the study specific data set can be retrieved from OSF (https://osf.io/q4pw3/ [embargo till 2021/12/31]). For this study, male participants were recruited via advertisements distributed on social media platforms such as Facebook and the study's webpage. Advertisements on social media platforms were restricted to men of 18 years or older in the countries Germany, 
Switzerland, Austria, Lichtenstein, Luxembourg, and Belgium. Aiming to recruit a large sample of men older than 18 years with sufficient German language skills to read and respond to the questionnaire in German language, all men irrespective of mental health status were eligible to participate. During the recruitment period from 15th March 2021 to 28th April 2021 a total number of 1087 people expressed interest in this study by visiting the starting page of the online questionnaire. A little more than half of the initially interested participants $(\mathrm{n}=597,54.92 \%)$ were not included in the final analyses for one of the following reasons: data privacy agreement not provided, declaration of consent not provided, self-reported insufficient German language skills, gender requirements not fulfilled, age of minority and/or incomplete data in the questionnaires. This resulted in a total number of 490 participants included in the analyses. Figure 1 presents participant flow. After providing written informed consent at the beginning of the survey, participants agreed to the data privacy statement and then went on to answer sociodemographic questions, COVID-19-related questions, and a set of psychometric instruments. For the subsequently described study, the average completion time was 20 minutes. However, several participants went on to participate in further online experiments following the completion of initial questionnaires, which are beyond the scope of this report. Further information on the study is provided elsewhere (Walther, Grub, Tsar, et al., 2021) or on the preregistration in OSF (https://osf.io/q4pw3/ [embargo till 2021/12/31]).

\subsection{Instruments}

\subsubsection{Sociodemographics and COVID-19 related questions}

The online survey started with sociodemographic questions assessing sufficiency of German skills, gender, dimensional gender (positioning between the two poles $1=$ masculine, $10=$ feminine), age, height, weight, nationality, relationship status, sexual orientation, education, and the household's yearly gross income. Participants were further asked whether a validated test confirmed that they were currently infected with COVID-19 or whether a 
validated test has previously confirmed that they had contracted COVID-19 since the beginning of the pandemic (yes/no). If participants answered "yes" to this question, they were asked which symptoms from a given list they currently or previously suffered from due to COVID-19 infection (e.g., respiratory symptoms, cardiovascular symptoms, fatigue, fever, pain symptoms, post exertional malaise, cognitive symptoms, nausea, etc.). The list of symptoms was based on previous research investigating COVID-19 and long COVID symptoms (Sudre et al., 2021). Further descriptives are presented in Table 1.

\subsubsection{Bem Sex-Role Inventory - Masculinity (BSRI-M)}

Consisting of a masculinity and a femininity scale, the Bem Sex Role Inventory (Bem, 1974) measures masculinity and femininity independently as a two-dimensional construct. Each scale contains personality characteristics or traits that were selected as masculine or feminine based on sex-typed social desirability (Bem, 1974). In the present study, a validated German version of the 30-item short version scale was used (Troche \& Rammsayer, 2011). To identify the degree of self-identified masculine gender orientation, the masculinity subscale BSRI-M was used (Cronbach's $\alpha=.83$; Troche $\&$ Rammsayer, 2011). The participants had to specify for each of the 15 items how well the items matched their selfperception on a seven-point Likert scale ranging from 1 (never of almost never true) to 7 (always or almost always true). Cronbach's $\alpha$ in the current study was .90 .

\subsubsection{Male Role Norm Inventory - Short Form (MRNI-SF)}

The Male Role Norms Inventory - Short Form (MRNI-SF; Levant et al., 2013) consists of 21 items and measures traditional masculine ideology with seven subscales. The participant indicates the degree to which he agrees with traditional masculinity ideology statements on a seven-point Likert scale $(1=$ strongly disagree, $7=$ strongly agree $)$. The seven subscales of the MRNI-SF represent the following dimensions: restrictive emotionality, self- 
reliance through mechanical skills, negativity toward sexual minorities, avoidance of femininity, importance of sex, dominance, toughness. A Cronbach's alpha of $\alpha=.92$ for men has been reported for the original English version of the MRNI-SF (Levant et al., 2013). Cronbach's $\alpha$ in the current study: MRNI-SF total score $=.94$, MRNI-SF negativity toward sexual minorities $=.87$, MRNI-SF restrictive emotionality $=.74$, MRNI-SF self-reliance through mechanical skills $=.88$, MRNI-SF avoidance of femininity $=.88$, MRNI-SF importance of $\operatorname{sex}=.88, \mathrm{MRNI}-\mathrm{SF}$ dominance $=.88, \mathrm{MRNI-SF}$ toughness $=.81$.

\subsubsection{Gender Role Conflict Scale - Short Form (GRCS-SF)}

The Gender Role Conflict Scale (O’Neil et al., 1986) measures patterns of gender role conflicts. The Gender Role Conflict Scale-Short Form (Wester et al., 2012) measures the following four patterns of gender role conflict: 1) success, power, and competition (SPC), 2) restrictive emotionality (RE), 3) restrictive affectionate behavior between men (RABBM), and 4) conflict between work and family relations (CBWFR). Consisting of a total number of 16 items, four items are dedicated to each pattern of GRC. The participants indicate the degree of experienced conflict on a six-point Likert scale $(0=$ strongly disagree, $5=$ strongly agree). Wester and colleagues (Wester et al., 2012) reported internal consistencies of $\alpha=.77$ .80. The German version of the Gender Role Conflict Scale - Short Form (Komlenac et al., 2018) was used in the present study. Cronbach's $\alpha$ in the current study was at .79 .

\subsubsection{Patient Health Questionnaire-9 (PHQ-9)}

The Patient Health Questionnaire (PHQ-9; Kroenke et al., 2001) assesses nine symptoms of major depressive disorder specified by the Diagnostic and Statistical Manual of Mental Disorders 5 (DSM-5; American Psychiatric Association, 2013). For each of the nine symptoms the participants rate how often they appeared within the preceding two-week period on a four-point Likert scale ranging from 0 (not at all) to 3 (almost every day). The 
PHQ-9 is applied in criteria-based diagnoses of depressive disorders with a cut-off $\geq 10$ within research and clinical practice (Levis et al., 2020) and has been shown to provide a reliable and valid measure of depression severity (Kroenke et al., 2001). In this study a German version of the PHQ-9 was used, which has been previously validated on a representative German-speaking sample (Cronbach's $\alpha=.89$; Martin et al., 2006). Cronbach's $\alpha$ in the current study was at .90 .

\subsubsection{Male Depression Risk Scale-22 (MDRS-22)}

The Male Depression Risk Scale (MDRS-22; Rice et al., 2013) consists of 22 items assessing externalizing depressive symptoms. For each item, the participant rates how often the symptom appeared within the preceding month on an eight-point Likert scale ranging from 0 (not at all) to 7 (almost always). The scale consists of six domains and considers gender-specific externalizing symptoms of depression and assesses them within the context of cultural norms related to masculinity. The six domains of the MDRS-22 include emotion suppression (i.e., "I bottled up my negative feelings"), drug use (i.e., "I used drugs to cope"), alcohol use (i.e., "I needed to have easy access to alcohol"), anger and aggression (i.e., "I overreacted to situations with aggressive behaviors"), somatic symptoms (i.e., "I had regular headaches"). In the present study the validated German version of MDRS-22 was used (Guttman's $\lambda_{2}=.62-.91$; Walther, Grub, Ehlert, et al., 2021). Cronbach's $\alpha$ in the current study was at 0.88 .

\subsubsection{Fear of COVID-19 Scale (FCV-19S) and COVID-19 Pandemic Stress Scale (CPSS)}

As measures for the evaluation of discriminant validity with regard to depression measures, we included the German version of the fear of COVID-19 scale (Ahorsu et al., 2020) consisting of 7 items where participants have to indicate the extent to which they do agree with the statements on fear of COVID-19 on a five-point Likert scale $(1=$ do not agree 
at all; 5 = fully agree). Cronbach's $\alpha$ in the current study was at .85 . We further included the German version of the COVID-19 Pandemic Stress Scale consisting of 10 items on aspects and consequences of COVID-19 such as hygienic behavior rules, contact restrictions, or actual fear of COVID-19 infection (Werner et al., 2021). The participants had to rate how stressed or anxious they felt about these topics during the last 2 weeks on a four-point Likert scale $(0=$ not burdened at all, $4=$ very burdened $)$. Cronbach's $\alpha$ in the current study was at .74.

\subsection{Statistical Analysis}

The statistical analyses and computations were performed with the software R (R Core Team, 2020) and the supplementary packages "psych" (Revelle, 2020); calculating internal consistencies, effect sizes, and correlations), "car" (Fox \& Sanford, 2019); estimating variance inflation factors), "rcompanion" (Mangiafico, 2021); MLE estimation of goodness-of-fit indices for logistic regression models), and "ggplot2" (Wickham, 2016); data visualization). The subsamples used for the analyses described in the following were obtained by dividing the participants into two groups consisting of men who (previously or currently) tested positive for COVID-19 and men who never tested positive for COVID-19. While for the initial analyses a significance level of $\alpha=.05$ was used, a sequential Holm-Bonferroni correction for multiple testing was applied post-hoc to control for alpha inflation due to the multiple comparisons with identical statistical endpoints (Holm, 1979). Additionally, due to the a-priori formulated hypothesis, one-sided hypothesis tests were used in all the analyses involving traditional masculinity constructs (BSRI, MRNI, GRCS, including all their subscales). Lastly, statistical assumptions were assessed using Levene's test for homogeneity of variance (Levene, 1961) for the $t$-tests and the generalized variance inflation factor (Fox \& Monette, 1992) as well as Cook's distance (Cook, 1977) for the logistic regression models. 
Initially, sample characteristics were obtained by calculating mean scores and frequency distributions for the total sample as well as for the two subgroups separately. Additional $t$ - and $\chi^{2}$-tests were then used to test for statistically significant group differences between the two subgroups. Secondly, correlational analyses were conducted by calculating Pearson's correlation coefficient for the relevant variables and subsequently testing their significance with two-sided $t$-tests. Thirdly, logistic regression analyses were performed to assess the predictive value of depressive symptoms, traditional masculinity, fear of COVID19 and COVID-19 pandemic related stress. These regression models included the participant's age, income, education level, and sexual orientation as covariates. In a last part, a more explorative approach was used to assess a possible association between traditional masculinity and COVID-19 symptoms. For this purpose, men who have contracted COVID-19 were divided into two subgroups using median division of the variables assessing the traditional masculinity constructs so that men with high and low traditional masculinity could be identified and compared with regard to displaying specific COVID-19 symptoms. Wald-tests were then used to determine significant differences in the frequency of COVID-19 symptoms and t-tests were used to determine significant differences in the mean scores of the remaining questionnaires used in the study.

\section{Results}

\subsection{Descriptive Statistics and Group Differences in Men with and without COVID-19}

\section{Infection}

Out of the 490 men participating in the survey, 34 (6.9\%) previously or currently tested positive for COVID-19, which corresponded to the COVID-19 positive rates at that time for the surveyed countries Germany (4.1\%), Austria (6.9\%), and Switzerland (1.5\%). Their age ranged from 18 to 68 years old, with a mean age of 25.7 years. The majority of the participants originated from Germany $(73.1 \%)$ and Switzerland (14.5\%), self-identified as 
heterosexual (73.7\%), were single (63.5\%), and had completed secondary education $(71.0 \%)$. More detailed sample demographics can be found in Table 1.

Regarding group differences (Figure 2, S-Table 1), men who have contracted COVID19 had significantly higher MRNI scores on the total scale (Cohen's $d=.44$ ) as well as on the MRNI subscales assessing Restrictive Emotionality (RE; $d=.38$ ), Importance of Sex (IS; $d=$ $.31)$, Dominance ( $\mathrm{D} ; d=.55)$, and Toughness $(\mathrm{T} ; d=.30)$. Similarly, men who had contracted COVID-19 also had significantly higher GRCS scores on the total scale $(d=.40)$ as well as on the subscale measuring Success, Power, Competition (SPC; $d=.38$ ). However, none of these effects remained significant after applying the Holm-Bonferroni correction for multiple testing.

\subsection{Correlational Analysis}

As presented in Table 2, the PHQ-9 was significantly positively correlated with the MDRS-22 $(r=.64)$, the GRCS $(r=.41)$, the FCV-19S $(r=.29)$, and the CPSS $(r=.31)$, while also being significantly negatively correlated with the BSRI $(r=-.36)$. The MDRS-22 was significantly positively correlated with the MRNI $(r=.18)$, the GRCS $(r=.38)$, the FCV-19 $(r=.28)$, and the CPSS $(r=.28)$. The BSRI was only significantly positively correlated with the MRNI $(r=.33)$ while also being significantly negatively correlated with the FCV-19S $(r=$ -.16). The MRNI was again significantly positively correlated with the GRCS $(r=.39)$ and significantly negatively correlated with the FCV-19S $(r=-.15)$. The GRCS was significantly positively correlated with the FCV-19S $(r=.18)$ and the CPSS $(r=.24)$. Lastly, the FCV-19S was significantly positively correlated with the CPSS $(r=.58)$.

\subsection{Logistic Regression predicting COVID-19 Infection}

For the binary logistic regression analysis, two models were fitted due to singularities in the covariance matrices caused by perfect linear combination among the MRNI total score 
and its subscales, as well as the GRCS and its subscales. The first model therefore included the total scores of the MRNI and the GRCS as predictors for a COVID-19 infection (S-Figure 1, S-Table 2) and revealed the PHQ-9 $(\mathrm{OR}=0.91[0.83-0.99]$, standardized OR $=0.53[0.30$ - 0.93]) and the MDRS-22 (OR $\left.=1.03[1.00-1.05], \mathrm{OR}_{\text {std. }}=1.66[1.04-2.65]\right)$ to be the only two significant predictors for a COVID-19 infection. The second model included only the subscales of the MRNI and the GRCS instead of the total scores, but still the same two predictors emerged as significant $\left(\mathrm{PHQ}-9: \mathrm{OR}=0.89[0.81-0.98], \mathrm{OR}_{\text {std. }}=0.47[0.25-\right.$ $0.85]$; MDRS-22: OR $\left.=1.02[1.00-1.05], \mathrm{OR}_{\text {std. }}=1.64[1.01-2.66]\right)$. Both models therefore indicate higher PHQ-9 scores to be associated with a lower likelihood to have contracted COVID-19 and higher MDRS-22 scores to be associated with a higher likelihood to have contracted COVID-19. However, these results also became non-significant after applying the Holm-Bonferroni correction for multiple testing. Nonetheless, further post-hoc analyses favored the first model with the total scores of the MRNI and the GRCS (BIC $=297.7, \chi^{2}(11)$ $=23.7, \mathrm{p}=.015)$ as a better overall fit in predicting a COVID-19 infection as compared to the second model including only the subscales of the MRNI and the GRCS (BIC $=349.3, \chi^{2}(20)$ $=27.8, \mathrm{p}=.114)$.

\subsection{Group Differences in Men with low and high Traditional Masculinity}

An explorative analysis was taken to further examine possible associations between high traditional masculinity, COVID-19 symptoms and depressive symptomatology in men who contracted COVID-19. Here, only the subsample consisting of 34 men was used. As can be seen in Figure 4A, men with high traditional masculinity operationalized as high BSRI scores exhibited less general (i.e., pain symptoms, headache, joint pain, muscle pain, peripheral neuropathy) pain symptomatology (47.4\%) as compared to men with low traditional masculinity $(80.0 \%)\left(\chi^{2}(1)=4.22, p=.040, \mathrm{OR}_{\text {std. }}=0.17[0.03-0.84]\right)$ and lower PHQ-9 scores $(M=9.2, S D=5.7)$ as compared to men with low traditional masculinity $(M=$ 
13.9, $S D=6.3)(t(32)=-2.26, \mathrm{p}=.031, d=.37)$. Furthermore, as can be seen in Figure 4C, men who experienced higher gender role conflict exhibited higher MDRS-22 scores $(M=$ $44.5, S D=21.5)$ as compared to men who experience lower gender role conflict $(M=23.8$, $S D=23.1)(t(32)=2.70, \mathrm{p}=.011, d=.43)$. However, these results also faded after applying the correction for multiple testing.

\section{Discussion}

\subsection{Summary of results}

The present study examined the interaction between mental health outcomes, traditional masculinity and COVID-19 infection. The first set of hypotheses postulated that higher expression of traditional masculinity constructs (gender role orientation, traditional masculine ideologies, gender role conflict) would be associated with a higher likelihood of having contracted COVID-19. Although group comparisons between men infected with COVID-19 and those without infection showed that infected men exhibit significantly elevated scores of traditional masculine ideologies and gender role conflict (but not gender role orientation), this could not be confirmed in the logistic regression models controlling for the confounders of age, income, education, and sexual orientation. Notably, when applying correction for multiple testing, the significant group differences did not hold.

In a second step the hypotheses that men with elevated prototypical depression symptoms would have a lower risk of being infected with COVID-19, whereas men with elevated male-typical externalizing depression symptoms would have an increased likelihood of being infected with COVID-19 were tested. Although this tendency was observable in the group comparisons, no significant group differences emerged. However, these hypotheses were confirmed subsequently in the logistic regression analyses controlling for confounders. It is important to note that when the Holm-Bonferroni correction for multiple testing was applied, the significant effects did not hold. 


\subsection{Integration of findings}

The results partly support our assumption that higher expression of traditional masculinity (MRNI-SF and GRCS-SF but not BSRI-M) is associated with a higher likelihood of having contracted COVID-19. Two out of the three masculinity measures were significantly elevated in the group of men reporting having contracted COVID-19 since the beginning of the pandemic as compared to men without known COVID-19 infection. Specifically, for endorsement of traditional masculine ideologies (MRNI-SF), the subscales of restrictive emotionality, importance of sex, dominance, and toughness were elevated in the men with a positive COVID-19 test, while for gender role conflict (GRCS-SF), the subscales of power, competition, and success were elevated. These findings are consistent with previous studies showing that adherence to traditional masculine norms is associated with engagement in risky health behaviors in men (Iwamoto et al., 2011; Levant \& Wimer, 2014). The present study thereby partly corroborates previous literature on traditional masculinity and health behaviors in general, but also with regard to mask wearing during the current pandemic (Mahalik et al., 2021); extending it with regard to the most critical outcome to fight the current Corona pandemic, namely contracting COVID-19.

Importantly, a non-peer reviewed report examining over 6,000 adults suggested that men self-identifying as "completely masculine" were almost three times more likely to contract COVID-19 than men self-identifying "mostly masculine" or "slightly masculine" (Cassino et al., 2021). Data stemming from the present study could not confirm such a direct association for any of the three validated masculinity measures highlighting three potential explanations for the observed lack of direct association. Firstly, it could be that included confounders (age, education, income, sexual orientation) better explain COVID-19 infection risk than traditional masculinity. Secondly, the study might have been underpowered to detect a signal due to the number of positive COVID-19 infection cases. Thirdly, it is imaginable 
that using validated masculinity scales reveal a more complex picture than reported by Cassino et al. (2021) using a single item to measure masculinity. This is in line with a previous report suggesting that total scores obscure the complex relation between several traditional masculine norms and health behaviors or outcomes (Gerdes \& Levant, 2018). This study further highlighted that in using the CMNI with its subscales as a measure for conformity to traditional masculinity, $30 \%$ of the findings reflected beneficial associations with specific masculine role norms and health or well-being outcomes, especially for health promotion. Therefore, the observed findings of uniformly increased traditional masculinity in the group having contracted COVID-19 (see Figure 2) are pointing towards a relation between traditional masculinity ideology and COVID-19 infection risk, although the Holm-Bonferroni correction renders the effects null. A further study from our workgroup is currently underway with the goal of replicating the observed findings with the advantage of accessing a larger population of contracted individuals with less time restrictions for recruitment due to the largely stabilized pandemic situation.

The fact that men with high traditional masculinity exhibit worse health behaviors including more risk-taking and less mask-wearing (Mahalik et al., 2021), and thus are exposed to a higher risk of infection, also further explains why men are more likely to die from COVID-19 as compared to women (Hamer et al., 2020; Peckham et al., 2020). The present study sheds light on why men, and particularly men with high traditional masculinity, may be at increased risk for contracting COVID-19, and thus potentially at increased mortality as shown in previous longitudinal studies (Coleman et al., 2020; Lippa et al., 2000). Furthermore, results supported the exploratory hypotheses that higher prototypical depression symptoms (PHQ-9) are associated with a reduced risk of contracting COVID-19, while higher male-typical externalizing depression symptoms (MDRS-22) are associated with an increased risk of contracting COVID-19. This finding is new insofar that no previous study has examined prototypical and male-typical externalizing depression symptoms in parallel 
with regard to the risk of contracting COVID-19. The observation that prototypical depression symptoms - probably due to their relation with a general social withdrawal - are associated with a reduced infection risk, has previously been reported by independent studies (Ceban et al., 2021a). However, the contrary or a null association has also been reported several times (Chang et al., 2020; Tang et al., 2020; Taquet et al., 2021; Wang et al., 2021), suggesting that this relationship is either non-existent or depends on examined subgroups and the specific depression symptomatology exhibited. A previous study from our workgroup examining adult men's psychotherapy use found that only higher male-typical externalizing depression symptoms, but not prototypical depression symptoms, predicted lower psychotherapy use (Eggenberger et al., 2021). Similarly, male-typical externalizing and prototypical depression symptoms were shown to differ in predicting past-month suicide attempt (Rice et al., 2019; Walther, Grub, Tsar, et al., 2021). These findings support the notion that internalizing and externalizing psychopathology are differentially related to health behavior risk and specifically to the risk of contracting COVID-19. Yet it is also relevant that COVID-19 increased the risk of suffering from depression measured with the PHQ-9 more than 3-fold (Ettman et al., 2020), which additionally highlights the importance to understand which individuals suffer from which depression symptoms and whether they are at particular risk of contracting COVID-19.

In summary, the present data support the hypothesis that traditional masculinity and the presence of male-typical externalizing depression symptoms may be associated with an increased risk of contracting COVID-19. Therefore, health promotion campaigns should specifically target these men, as they might be relatively poorly informed due to a more indifferent attitude based in traditional male role norms of toughness and self-reliance, or in extreme cases these men may also deliberately infect themselves with COVID-19 to self-harm or suicide (Ammerman et al., 2021). One way to address this problem would be to appeal to the role of protector and provider and ask men with high traditional masculinity to protect 
others and not put them in danger, which also means protecting themselves so as not to be carriers of the virus.

Mental health care specialists should pay particular attention to men with high traditional masculinity and male-typical externalizing depression symptoms and inform them about the detrimental personal and societal consequences a careless spreading of the virus has. The effect men with high traditional masculinity and male-typical externalizing depression symptoms have in spreading the virus and thereby prolonging the current pandemic situation is difficult to identify. Nevertheless, informing men and the entire health care system about the crucial role men with high traditional masculinity may play in this pandemic is of vital importance. However, the use of a strength-based approach by appealing to the role of protector and provider or good leader may be all the more promising in limiting the risk of COVID-19 infection by men with high traditional masculinity as a means of protecting significant others.

It is important to note, however, that the present findings can only be considered preliminary results, since limited power led significant results to fade after correction for multiple testing. Due to a time-sensitive measurement period in the COVID-19 pandemic, it was not possible to recruit participants for a sufficiently long period and therefore only a small sample of 490 men in total and 34 currently or previously COVID-19 infected men could be obtained. Therefore, the present findings based on the a priori formulated hypotheses are to be considered tentative. The observation that findings uniformly fade when applying correction for multiple testing is, however, also to be interpreted with caution since no prior results on this specific research question is available and potentially a substantially larger sample size would be required to uphold the small to moderate findings in the range of statistical significance due to small number of positive COVID-19 cases. Due to the time restrictions set by a dynamic pandemic situation, this poses a major challenge for research and thus corrections for multiple testing must be placed in perspective. 
Of further interest are the observations regarding traditional masculinity and specific COVID-19-related symptoms (see Figure 4). A detailed discussion of these findings is provided in the Supplementary.

\subsection{Limitations}

When interpreting the study results, some limitations should be taken into account. First, the cross-sectional design only provides information about associations and no causal conclusions can be drawn from the results. Furthermore, the sample with a total of 490 men and only 34 men with a reported COVID-19 infection is not sufficiently powered to identify small effects. Therefore, all significant results faded after the Holm-Bonferroni correction. Furthermore, the main outcome measure of a positive corona infection by a validated test has been selfreported, and because of insufficient data, little can be said about its reliability despite its high face validity. It also becomes evident from the descriptive data showing a high percentage of men suffering from a psychological disorder or suicidal thoughts, that this is a sample of adult men with high psychological distress, which makes it difficult to generalize to the male population as a whole. Lastly, it should be noted that the PHQ-9 and MDRS-22 depression scales, while very well validated measures of depression, are not capable of identifying depressive disorders and thus interpretations regarding depression status and risk for contracting COVID-19 in men need to be made on syndrome level, while a clinical diagnostic interview enabling differential diagnosis would be preferable.

\subsection{Conclusion}

Taken together, men exhibiting higher traditional masculinity and higher male-typical externalizing depression symptoms may be at increased risk for contracting COVID-19 and should be informed by health care campaigns and mental health care specialists. Appealing to these men's provider and protector role might partially counter the overall detrimental effects 
of high traditional masculinity without challenging the endorsement of traditional masculinity ideology itself. The potential effect men with high traditional masculinity and increased maletypical externalizing depression symptoms have in prolonging the current pandemic is difficult to identify. More research using validated traditional masculinity measures combined with larger samples including more men with current or past COVID-19 infections is needed.

\section{Acknowledgements}

We thank all the participants for completing the survey and thereby supporting men's mental health research.

\section{Author contribution}

Design and conceptualization of the study: AW, JG, UE; Participant recruitment and testing: AW, JG; Data analysis and visualization: LE, AW; Data interpretation: AW, LE; Writing of the first draft: AW, LE; Editing of subsequent versions: AW, UE, ZES, SMR, DK, JSO, JLO.

\section{Conflict of interest statement}

The authors declare no conflict of interest.

\section{Funding information}

There was no specific funding for this research. 


\section{References}

Ahorsu, D. K., Lin, C.-Y., Imani, V., Saffari, M., Griffiths, M. D., \& Pakpour, A. H. (2020). The Fear of COVID-19 Scale: Development and Initial Validation. International Journal of Mental Health and Addiction. https://doi.org/10.1007/s11469-020-00270-8

American Psychiatric Association. (2013). Diagnostic and statistical manual of mental disorders (DSM-5®) (5th ed.). American Psychiatric Pub.

Ammerman, B. A., Burke, T. A., Jacobucci, R., \& McClure, K. (2021). Preliminary investigation of the association between COVID-19 and suicidal thoughts and behaviors in the U.S. Journal of Psychiatric Research, 134(December 2020), 32-38.

https://doi.org/10.1016/j.jpsychires.2020.12.037

Babalola, S., Krenn, S., Rimal, R., Serlemitsos, E., Shaivitz, M., Shattuck, D., \& Storey, D. (2020). KAP COVID Dashboard. Johns Hopkins Center for Communication Programs, Massachusetts Institute of Technology, Global Outbreak Alert and Response Network, Facebook Data for Good.

Bem, S. L. (1974). The measurement of psychological androgyny. Journal of Consulting and Clinical Psychology, 42(2), 155-162. https://doi.org/10.1037/h0036215

Bridges, T., Barber, K., Nelson, J. D., \& Chatillon, A. (2021). Masculinity and COVID-19: Symposium Introduction. Men and Masculinities, 24(1), 163-167. https://doi.org/10.1177/1097184X211004325

Capraro, V., \& Barcelo, H. (2020). The effect of messaging and gender on intentions to wear a face covering to slow down COVID-19 transmission. PsyArXiv. https://doi.org/10.31234/osf.io/tg7vz

Cassino, D., \& Besen-Cassino, Y. (2020). Of Masks and Men? Gender, Sex, and Protective Measures during COVID-19. Politics \& Gender, 16(4), 1052-1062. https://www.fdu.edu/news/fdu-pollfinds-masculinity-is-a-major-risk-factor-for-covid-19/

Cavanagh, A., Wilson, C. J., Kavanagh, D. J., \& Caputi, P. (2017). Differences in the Expression of Symptoms in Men Versus Women with Depression: A Systematic Review and Meta-analysis. Harvard Review of Psychiatry, 25(1), 29-38. https://doi.org/10.1097/HRP.0000000000000128

Ceban, F., Nogo, D., Carvalho, I. P., Lee, Y., Nasri, F., Xiong, J., Lui, L. M. W., Subramaniapillai, M., Gill, H., \& Liu, R. N. (2021a. Association Between Mood Disorders and Risk of COVID-19 Infection, Hospitalization, and Death: A Systematic Review and Meta-analysis. JAMA Psychiatry.

Chang, T. S., Ding, Y., Freund, M. K., Johnson, R., Schwarz, T., Yabu, J. M., Hazlett, C., Chiang, J. N., Wulf, A., \& Geschwind, D. H. (2020). Prior diagnoses and medications as risk factors for COVID-19 in a Los Angeles Health System. MedRxiv.

Clark, C., Davila, A., Regis, M., \& Kraus, S. (2020). Predictors of COVID-19 voluntary compliance behaviors: An international investigation. Global Transitions, 2, 76-82.

Coleman, D., Feigelman, W., \& Rosen, Z. (2020). Association of High Traditional Masculinity and Risk of Suicide Death: Secondary Analysis of the Add Health Study. JAMA Psychiatry, 77(4), 435-437. https://doi.org/10.1001/jamapsychiatry.2019.4702

Cook, R. D. (1977). Detection of influential observation in linear regression. Technometrics, 19(1), 15-18. https://doi.org/10.1080/00401706.1977.10489493

Courtenay, W. H. (2000). Engendering Health: A Social Constructionist Examination of Men's Health Beliefs and Behaviors. Psychology of Men and Masculinity, 1(1), 4-15.

https://doi.org/10.1037/1524-9220.1.1.4 
Eggenberger, L., Fordschmid, C., Ludwig, C., Weber, S., Grub, J., Komlenac, N., \& Walther, A. (2021). Men's Psychotherapy Use, Male Role Norms, and Male-Typical Depression Symptoms: Examining 716 Men and Women Experiencing Psychological Distress. Behavioral Sciences, 11(6), 83 .

Ettman, C. K., Abdalla, S. M., Cohen, G. H., Sampson, L., Vivier, P. M., \& Galea, S. (2020). Prevalence of depression symptoms in US adults before and during the COVID-19 pandemic. JAMA Network Open, 3(9), e2019686-e2019686.

Fox, J., \& Monette, G. (1992). Generalized collinearity diagnostics. Journal of the American Statistical Association, 87(417), 178-183. https://doi.org/10.1080/01621459.1992.10475190

Fox, J., \& Sanford, W. (2019). An $\{R\}$ Companion to Applied Regression, Third Edition. Sage.

Gerdes, Z. T., \& Levant, R. F. (2018). Complex Relationships Among Masculine Norms and Health/Well-Being Outcomes: Correlation Patterns of the Conformity to Masculine Norms Inventory Subscales. American Journal of Men's Health, 12(2), 229-240. https://doi.org/10.1177/1557988317745910

Haischer, M. H., Beilfuss, R., Hart, M. R., Opielinski, L., Wrucke, D., Zirgaitis, G., Uhrich, T. D., \& Hunter, S. K. (2020). Who is wearing a mask? Gender-, age-, and location-related differences during the COVID-19 pandemic. Plos One, 15(10), e0240785.

Hamer, M., Kivimäki, M., Gale, C. R., \& Batty, G. D. (2020). Lifestyle risk factors, inflammatory mechanisms, and COVID-19 hospitalization: A community-based cohort study of 387,109 adults in UK. Brain, Behavior, and Immunity, 87, 184-187.

Harrison, J. (1978). Warning: The male sex role may be dangerous to your health. Journal of Social Issues, 34(1), 65-86.

Holm, S. (1979). A Simple Sequentially Rejective Multiple Test ProcedureAuthor. Scandinavian Journal of Statistics, 6(2), 65-70.

Iwamoto, D. K., Cheng, A., Lee, C. S., Takamatsu, S., \& Gordon, D. (2011). "Man-ing” up and getting drunk: The role of masculine norms, alcohol intoxication and alcohol-related problems among college men. Addictive Behaviors, 36(9), 906-911.

Izurieta, H. S., Graham, D. J., Jiao, Y., Hu, M., Lu, Y., Wu, Y., Chillarige, Y., Wernecke, M., Menis, M., \& Pratt, D. (2021). Natural History of Coronavirus Disease 2019: Risk Factors for Hospitalizations and Deaths Among> 26 Million US Medicare Beneficiaries. The Journal of Infectious Diseases, 223(6), 945-956.

Jeon, H.-L., Kwon, J. S., Park, S.-H., \& Shin, J.-Y. (2021). Association of mental disorders with SARS-CoV-2 infection and severe health outcomes: nationwide cohort study. The British Journal of Psychiatry, 218(6), 344-351.

Komlenac, N., Siller, H., Bliem, H. R., \& Hochleitner, M. (2018). Validation of the internal structure of a German-language version of the gender role conflict scale - Short form. Frontiers in Psychology, 9(JUL), 1-5. https://doi.org/10.3389/fpsyg.2018.01161

Kroenke, K., Spitzer, R. L., \& Williams, J. B. W. (2001). The PHQ-9: validity of a brief depression severity measure. Journal of General Internal Medicine, 16(9), 606-613.

Levant, R. F., Hall, R. J., \& Rankin, T. J. (2013). Male Role Norms Inventory-Short Form (MRNI$\mathrm{SF})$ : Development, confirmatory factor analytic investigation of structure, and measurement invariance across gender. Journal of Counseling Psychology, 60(2), 228. 
Levant, R. F., Hirsch, L. S., Celentano, E., \& Cozza, T. M. (1992). The male role: An investigation of contemporary norms. Journal of Mental Health Counseling.

Levant, R. F., \& Richmond, K. (2015). The gender role strain paradigm and masculinity ideologies. APA Handbook of Men and Masculinities., 23-49. https://doi.org/10.1037/14594-002

Levant, R. F., \& Wimer, D. J. (2014). Masculinity Constructs as Protective Buffers and Risk Factors for Men's Health. American Journal of Men's Health, 8(2), 110-120. https://doi.org/10.1177/1557988313494408

Levene, H. (1961). Robust tests for equality of variances. Contributions to Probability and Statistics. Essays in Honor of Harold Hotelling, 279-292.

Levis, B., Sun, Y., He, C., Wu, Y., Krishnan, A., Bhandari, P. M., Neupane, D., Imran, M., Brehaut, E., Negeri, Z., Fischer, F. H., Benedetti, A., \& Thombs, B. D. (2020). Accuracy of the PHQ-2 Alone and in Combination with the PHQ-9 for Screening to Detect Major Depression: Systematic Review and Meta-analysis. JAMA - Journal of the American Medical Association, 323(22), 2290-2300. https://doi.org/10.1001/jama.2020.6504

Li, M., Zhang, Z., Cao, W., Liu, Y., Du, B., Chen, C., Liu, Q., Uddin, M. N., Jiang, S., Chen, C., Zhang, Y., \& Wang, X. (2021). Identifying novel factors associated with COVID-19 transmission and fatality using the machine learning approach. Science of the Total Environment, 764. https://doi.org/10.1016/j.scitotenv.2020.142810

Lippa, R. A., Martin, L. R., \& Friedman, H. S. (2000). Gender-related individual differences and mortality in the terman longitudinal study: Is masculinity hazardous to your health? Personality and Social Psychology Bulletin, 26(12), 1560-1570. https://doi.org/10.1177/01461672002612010

Mahalik, J. R., Bianca, M. di, \& Harris, M. P. (2021). Men's attitudes toward mask-wearing during COVID-19: Understanding the complexities of mask-ulinity. Journal of Health Psychology. https://doi.org/10.1177/1359105321990793

Mahalik, J. R., Locke, B. D., Ludlow, L. H., Diemer, M. A., Scott, R. P. J., Gottfried, M., \& Freitas, G. (2003). Development of the conformity to masculine norms inventory. Psychology of Men \& Masculinity, 4(1), 3.

Mangiafico, S. (2021). rcompanion: Functions to support extension education program evaluation (2.4.0).

Martin, A., Rief, W., Klaiberg, A., \& Braehler, E. (2006). Validity of the brief patient health questionnaire mood scale (PHQ-9) in the general population. General Hospital Psychiatry, 28(1), $71-77$.

Martin, L. A., Neighbors, H. W., \& Griffith, D. M. (2013). The experience of symptoms of depression in men vs women: Analysis of the national comorbidity survey replication. JAMA Psychiatry, 70(10), 1100-1106. https://doi.org/10.1001/jamapsychiatry.2013.1985

Nemani, K., Li, C., Olfson, M., Blessing, E. M., Razavian, N., Chen, J., Petkova, E., \& Goff, D. C. (2021). Association of psychiatric disorders with mortality among patients with COVID-19. JAMA Psychiatry, 78(4), 380-386.

Okten, I. O., Gollwitzer, A., \& Oettingen, G. (2020). Gender differences in preventing the spread of coronavirus.

O'Neil, J. M. (2013). Gender role conflict research 30 years later: An evidence-based diagnostic schema to assess boys and men in counseling. Journal of Counseling and Development, 91(4), 490-498. https://doi.org/10.1002/j.1556-6676.2013.00122.x 
O’Neil, J. M., Helms, B. J., Gable, R. K., David, L., \& Wrightsman, L. S. (1986). Gender-Role Conflict Scale: College men's fear of femininity. Sex Roles, 14(5), 335-350.

Palmer, C. L., \& Peterson, R. D. (2020a). Toxic Mask-ulinity: The Link between Masculine Toughness and Affective Reactions to Mask Wearing in the COVID-19 Era. Politics and Gender, 16(4), 1044-1051. https://doi.org/10.1017/S1743923X20000422

Peckham, H., de Gruijter, N. M., Raine, C., Radziszewska, A., Ciurtin, C., Wedderburn, L. R., Rosser, E. C., Webb, K., \& Deakin, C. T. (2020a). Male sex identified by global COVID-19 metaanalysis as a risk factor for death and ITU admission. Nature Communications, 11(1), 1-10.

Pleck, J. H. (1981). The myth of masculinity. MIT Press (MA).

R Core Team. (2020). R: A language and environment for statistical computing (4.0.3). R Foundation for Statistical Computing, Vienna, Austria.

Revelle, W. (2020). psych: Procedures for personality and psychological research (2.1.3). Northwestern University, Evanston, Illinois, USA.

Rice, S. M., Fallon, B. J., Aucote, H. M., \& Möller-Leimkühler, A. M. (2013). Development and preliminary validation of the male depression risk scale: Furthering the assessment of depression in men. Journal of Affective Disorders, 151(3), 950-958.

https://doi.org/10.1016/j.jad.2013.08.013

Rice, S. M., Ogrodniczuk, J. S., Kealy, D., Seidler, Z. E., Dhillon, H. M., \& Oliffe, J. L. (2019). Validity of the Male Depression Risk Scale in a representative Canadian sample: sensitivity and specificity in identifying men with recent suicide attempt. Journal of Mental Health, 28(2), 132 140. https://doi.org/10.1080/09638237.2017.1417565

Salvatore, M., Gu, T., Mack, J. A., Prabhu Sankar, S., Patil, S., Valley, T. S., Singh, K., Nallamothu, B. K., Kheterpal, S., \& Lisabeth, L. (2021). A phenome-wide association study (PheWAS) of COVID-19 outcomes by race using the electronic health records data in Michigan Medicine. Journal of Clinical Medicine, 10(7), 1351.

Shinn, A. K., \& Viron, M. (2020). Perspectives on the COVID-19 pandemic and individuals with serious mental illness. The Journal of Clinical Psychiatry, 81(3), 0.

Sudre, C. H., Murray, B., Varsavsky, T., Graham, M. S., Penfold, R. S., Bowyer, R. C., Pujol, J. C., Klaser, K., Antonelli, M., \& Canas, L. S. (2021). Attributes and predictors of long COVID. Nature Medicine, 27(4), 626-631.

Tang, O., Bigelow, B. F., Sheikh, F., Peters, M., Zenilman, J. M., Bennett, R., \& Katz, M. J. (2020). Outcomes of nursing home COVID-19 patients by initial symptoms and comorbidity: results of universal testing of 1970 residents. Journal of the American Medical Directors Association, 21(12), 1767-1773.

Taquet, M., Luciano, S., Geddes, J. R., \& Harrison, P. J. (2021). Bidirectional associations between COVID-19 and psychiatric disorder: retrospective cohort studies of 62354 COVID-19 cases in the USA. The Lancet Psychiatry, 8(2), 130-140.

Troche, S., \& Rammsayer, T. (2011). Eine Revision des deutschsprachigen Bem Sex-Role Inventory. Klinische Diagnostik Und Evaluation, 4, 262-283.

Umamaheswar, J., \& Tan, C. (2020). "Dad, Wash Your Hands": Gender, Care Work, and Attitudes toward Risk during the COVID-19 Pandemic. Socius, 6, 2378023120964376.

Walther, A., Grub, J., Ehlert, U., Wehrli, S., Rice, S., Seidler, Z. E., \& Debelak, R. (2021). Male depression risk, psychological distress, and psychotherapy uptake: validation of the German 
version of the male depression risk scale. Journal of Affective Disorders Reports, 100107. https://doi.org/https://doi.org/10.1016/j.jadr.2021.100107

Walther, A., Grub, J., Tsar, S., Ehlert, U., ..., \& Eggenberger, L. (2021). Status loss due to COVID-19, traditional masculinity, and the prediction of suicidal ideation and recent suicide attempts. PsyArXiv.

Walther, A., \& Seidler, Z. E. (2020). Männliche Formen der Depression und deren Behandlung. PiDPsychotherapie Im Dialog, 21(04), 40-45. https://doi.org/10.1055/a-0987-5902

Wang, Q., Xu, R., \& Volkow, N. D. (2021). Increased risk of COVID-19 infection and mortality in people with mental disorders: analysis from electronic health records in the United States. World Psychiatry, 20(1), 124-130.

Werner, A., Kater, M.-J., Schlarb, A., \& Lohaus, A. (2021). Covid-19-pandemie-stress-skala (CPSS).

Wester, S. R., Vogel, D. L., O’Neil, J. M., \& Danforth, L. (2012). Development and evaluation of the Gender Role Conflict Scale Short Form (GRCS-SF). Psychology of Men \& Masculinity, 13(2), 199.

Wickham, H. (2016). ggplot2: Elegant graphics for data analysis. Springer-Verlag, New York.

Winkler, D., Pjrek, E., \& Kasper, S. (2005). Anger attacks in depression-evidence for a male depressive syndrome. Psychotherapy and Psychosomatics, 74(5), 303-307.

Wong, Y. J., Ho, M. H. R., Wang, S. Y., \& Miller, I. S. K. (2017). Meta-analyses of the relationship between conformity to masculine norms and mental health-related outcomes. Journal of Counseling Psychology, 64(1), 80-93. https://doi.org/10.1037/cou0000176

Yao, H., Chen, J. H., \& Xu, Y. F. (2020). Patients with mental health disorders in the COVID-19 epidemic. The Lancet Psychiatry, 7(4), e21. https://doi.org/10.1016/S2215-0366(20)30090-0 
Table 1. Descriptive Statistics for the Sample

\begin{tabular}{|c|c|c|c|c|c|c|c|c|}
\hline & \multicolumn{2}{|c|}{ Total $(N=490)$} & \multicolumn{2}{|c|}{ No CV19 $(N=456)$} & \multicolumn{2}{|c|}{$\operatorname{CV} 19(N=34)$} & \multirow[b]{2}{*}{ test-statistic $(d f)$} & \multirow[b]{2}{*}{$p$} \\
\hline & $N(\%)$ & $M(S D)$ & $N(\%)$ & $M(S D)$ & $N(\%)$ & $M(S D)$ & & \\
\hline Age & & $25.7(9.8)$ & & $25.7(9.8)$ & & $25.9(9.5)$ & $-0.10(448)$ & .924 \\
\hline Nationality & & & & & & & $26.63(6)$ & $<.001^{* * *}$ \\
\hline Swiss & $71(14.5)$ & & $62(13.6)$ & & $9(26.5)$ & & & \\
\hline German & $358(73.1)$ & & $342(75.0)$ & & $16(47.1)$ & & & \\
\hline Austrian & $43(8.8)$ & & $36(7.9)$ & & $7(20.6)$ & & & \\
\hline Luxembourger & $4(0.8)$ & & $4(0.9)$ & & $0(0)$ & & & \\
\hline Liechtensteiner & $1(0.2)$ & & $1(0.2)$ & & $0(0)$ & & & \\
\hline Belgian & $1(0.2)$ & & $0(0)$ & & $1(2.9)$ & & & \\
\hline Other & $12(2.4)$ & & $11(2.4)$ & & $1(2.9)$ & & & \\
\hline Sexual orientation & & & & & & & $0.88(4)$ & .927 \\
\hline Heterosexual-identified & $361(73.7)$ & & $334(73.2)$ & & $27(79.4)$ & & & \\
\hline Gay/Lesbian-identified & $39(8.0)$ & & $37(8.1)$ & & $2(5.9)$ & & & \\
\hline Bisexual-identified & $67(13.7)$ & & $63(13.8)$ & & $4(11.8)$ & & & \\
\hline Asexual-identified & $5(1.0)$ & & $5(1.1)$ & & $0(0)$ & & & \\
\hline Other & $18(3.7)$ & & $17(3.7)$ & & $1(2.9)$ & & & \\
\hline Marital status & & & & & & & $1.45(2)$ & .484 \\
\hline Single & $311(63.5)$ & & $291(63.8)$ & & $20(58.8)$ & & & \\
\hline In a relationship & $168(34.3)$ & & $154(33.8)$ & & $14(41.2)$ & & & \\
\hline $\begin{array}{l}\text { Separated after } \\
\text { permanent relationship }\end{array}$ & $11(2.2)$ & & $11(2.4)$ & & $0(0)$ & & & \\
\hline Education & & & & & & & 3.69 (3) & .296 \\
\hline None completed & $10(2.0)$ & & $10(2.2)$ & & $0(0)$ & & & \\
\hline Secondary education & $348(71.0)$ & & $325(71.3)$ & & $23(67.6)$ & & & \\
\hline Tertiary education & $106(21.6)$ & & $99(21.7)$ & & 7 (20.6) & & & \\
\hline Other & $26(5.3)$ & & $22(4.8)$ & & $4(11.8)$ & & & \\
\hline
\end{tabular}




\section{Yearly household income \\ (in CHF)}

$$
\begin{aligned}
& <25^{\prime} 000 \\
& 25^{\prime} 000-50^{\prime} 000
\end{aligned}
$$$$
>50 \text { '000 }
$$

\section{Due to CV19 Pandemic}

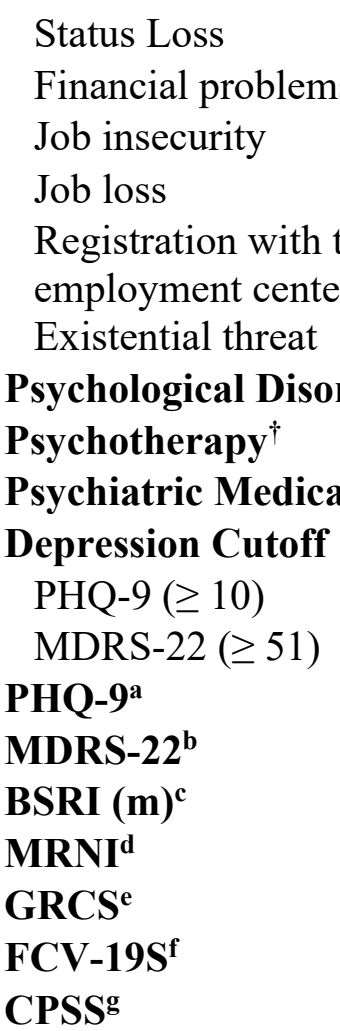

$\begin{array}{ll}233(47.6) & \\ 92(18.8) & \\ 165(33.7) & \\ 72(14.7) & \\ 100(20.4) & \\ 112(22.9) & \\ 41(8.4) & \\ 34(6.9) & \\ 95(19.4) & \\ 117(23.9) & \\ 95(19.4) & \\ 64(13.1) & \\ & \\ 322(65.7) & \\ 67(13.7) & \\ & 12.6(6.6) \\ & 29.2(20.6) \\ & 62.5(14.6) \\ & 45.6(21.4) \\ & 47.0(12.6) \\ & 12.8(4.9) \\ & 23.9(5.2)\end{array}$

$\begin{array}{cc}220(48.2) & 13(38.2) \\ 86(18.9) & 6(17.6) \\ 150(32.9) & 15(44.1)\end{array}$

$$
\begin{gathered}
67(14.7) \\
92(20.2) \\
102(22.4) \\
37(8.1)
\end{gathered}
$$$$
30(6.6)
$$$$
86 \text { (18.9) }
$$$$
112(24.6)
$$$$
92(20.2)
$$$$
62 \text { (13.6) }
$$

\section{5 (14.7)}

$8(23.5)$

10 (29.4)

$4(11.8)$

$\begin{array}{cc}0(1) & 1 \\ 0.06(1) & .804 \\ 0.54(1) & .464 \\ 0.18(1) & .674\end{array}$

4 (11.8)

0.64 (1)

$9(26.5)$

$5(14.7)$

$3(8.8)$

$2(5.9)$

0.74 (1)

$1.19(1)$

$1.93(1)$

1.05 (1)

.425

305 (66.9)

17 (50.0)

9 (26.5)

3.29 (1)

$$
3.07 \text { (1) }
$$$$
1.21(488)
$$

$11.3(6.3)$

$34.1(24.4)$

$-1.45(488)$

$28.8(20.3)$

$62.2(14.6)$

44.9 (20.7)

46.7 (12.2)

$12.9(4.90)$

65.4 (15.2)

$54.4(28.9)$

$51.7(16.0)$

$-1.20(488)$

-1.87 (35.6)

-1.78 (35.9)

$1.28(488)$

$22.6(5.38) \quad 1.52(488)$

.391

.275

.164

.306

.070

$.046 *$

.225

.148

.116

$.035 *$

$.042^{*}$

.200 .130

Note. $N=$ number of participants, $M=$ mean, $S D=$ standard deviation, test-statistic $=\mathrm{t}$-value for continuous, $\chi^{2}$-value for categorical variables, $d f=$ degrees of freedom, $p=p$-value, $C V 19=$ COVID-19. Subjective social status was dichotomized using a median-split.

$\dagger$ Assessed in self-report 
${ }^{\mathrm{a}}$ PHQ-9 = Patient Health Questionnaire $-9 ;{ }^{\mathrm{b}}$ MDRS-22 = Male Depression Risk Scale $-22 ;{ }^{\mathrm{c}}$ BSRI $(\mathrm{m})=$ Bem Sex-Role Inventory $(m=$ masculinity subscale); ${ }^{\mathrm{d}} \mathrm{MRNI}=$ Male Role Norms Inventory; ${ }^{\mathrm{e}}$ GRCS = Gender Role Conflict Scale; ${ }^{\mathrm{f}}$ FCV-19S = Fear of COVID-19 Scale; g CPSS = COVID-19 Pandemic Stress Scale

${ }^{*}=p<.05$ 
Table 2. Correlation Matrix for Study Variables

\begin{tabular}{|c|c|c|c|c|c|c|c|c|c|}
\hline & $M$ & $S D$ & 1 & 2 & 3 & 4 & 5 & 6 & \\
\hline 1. PHQ-9a & 12.6 & 6.6 & - & & & & & & \\
\hline 2. MDRS- $22^{\mathrm{b}}$ & 29.2 & 20.6 & $.64^{* * * *}$ & - & & & & & \\
\hline 3. BSRI $(\mathrm{m})^{\mathrm{c}}$ & 62.5 & 14.6 & $-.36^{* * *}$ & -.10 & - & & & & \\
\hline 4. $\mathrm{MRNI}^{\mathrm{d}}$ & 45.6 & 21.4 & -.06 & $.17^{* *}$ & $.33^{* * *}$ & - & & & \\
\hline 5. $\mathrm{GRCS}^{\mathrm{e}}$ & 47.0 & 12.6 & $.41^{* * *}$ & $.48^{* * * *}$ & .01 & $.39^{* * *}$ & - & & \\
\hline 6. FCV-19S & 12.8 & 4.9 & $.29^{* * * *}$ & $.28^{* * *}$ & $-.16^{* *}$ & $-.15^{* *}$ & $.18^{* * *}$ & - & \\
\hline 7. $\mathrm{CPSS}^{\mathrm{g}}$ & 23.9 & 5.2 & $.31^{* * * *}$ & $.28^{* * * *}$ & .03 & -.04 & $.24^{* * *}$ & $.58^{* * *}$ & - \\
\hline
\end{tabular}

Note. $M=$ mean, $S D=$ standard deviation. $p$-values were adjusted for multiple testing using the Holm-Bonferroni method.

${ }^{\mathrm{a}}$ PHQ-9 = Patient Health Questionnaire - 9; ${ }^{\mathrm{b}}$ MDRS-22 = Male Depression Risk Scale - 22; ${ }^{\mathrm{c}}$ BSRI (m) = Bem Sex-Role Inventory

(masculinity subscale); ${ }^{\mathrm{d}} \mathrm{MRNI}=$ Male Role Norms Inventory; ${ }^{\mathrm{e}} \mathrm{GRCS}=$ Gender Role Conflict Scale; ${ }^{\mathrm{f}}$ FCV-19S = Fear of COVID-19 Scale; g CPSS $=$ COVID-19 Pandemic Stress Scale.

${ }^{* *}=p<.01,{ }^{* * *}=p<.001$ 


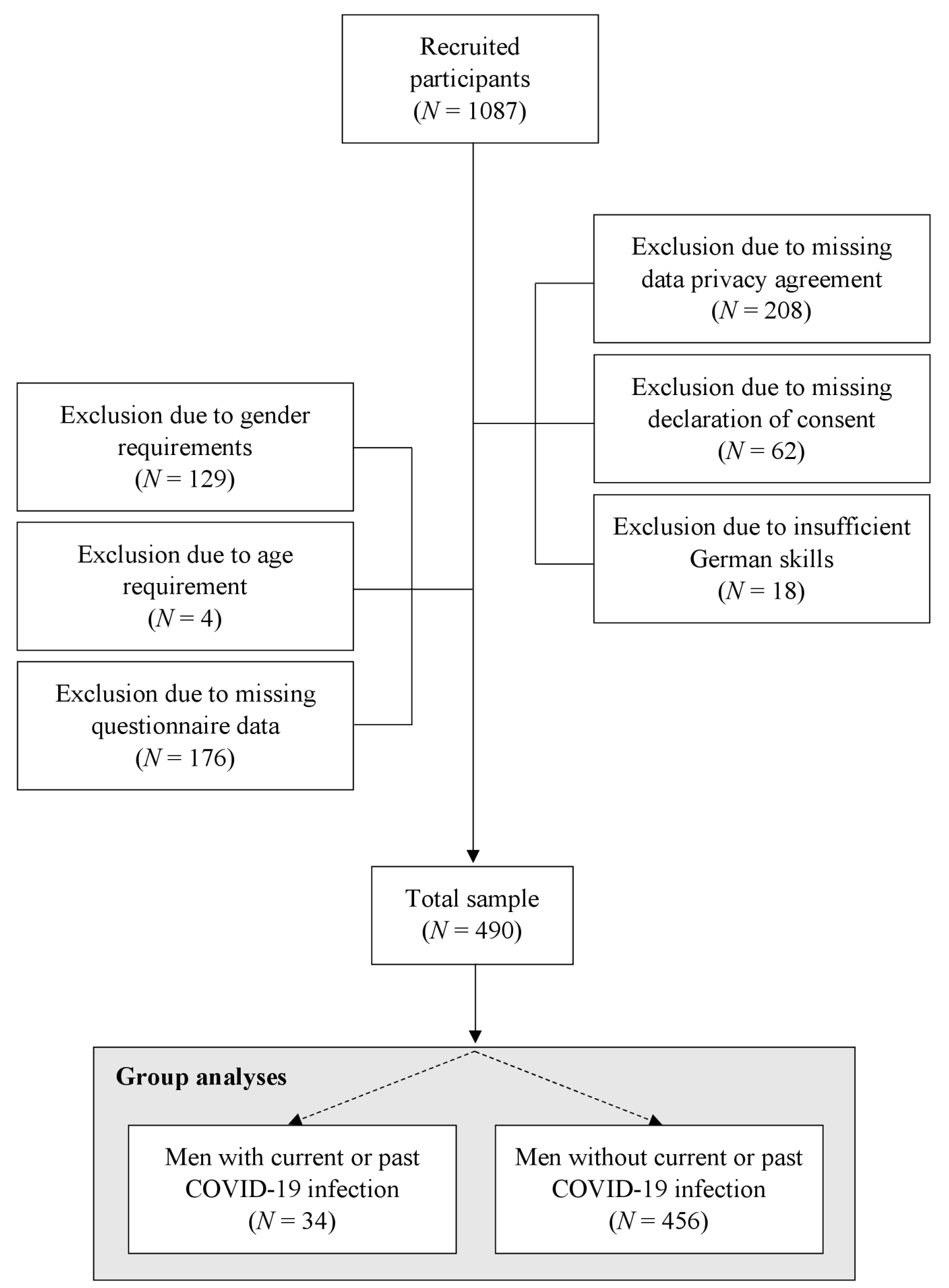

Figure 1. Flow diagram of the inclusion and exclusion process

Note. $N=$ number of participants 


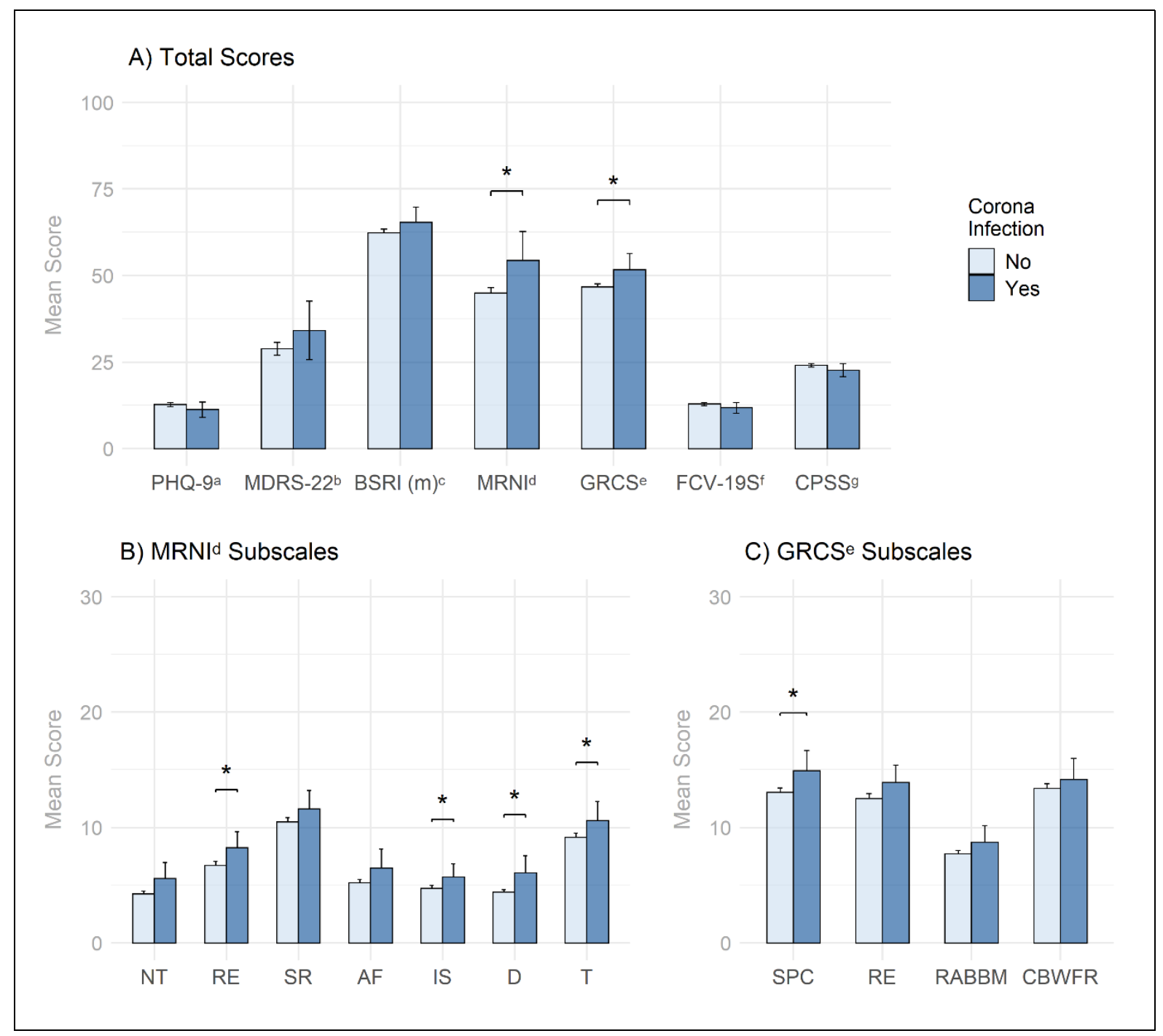

Figure 2. Mean Score Comparisons between Men with and without COVID-19 Infection and their two- and one-sided 95\% Confidence Intervals.

Note. ${ }^{\text {a }}$ PHQ-9 = Patient Health Questionnaire - 9; ${ }^{\mathrm{b}}$ MDRS-22 = Male Depression Risk Scale $-22 ;{ }^{c}$ BSRI $(\mathrm{m})=$ Bem Sex-Role Inventory (masculinity subscale); ${ }^{\mathrm{d}} \mathrm{MRNI}=$ Male Role Norms Inventory (subscales: NT = Negativity toward Sexual Minorities; RE $=$ Restrictive Emotionality; SR = Self-reliance through Mechanical Skills; AF = Avoidance of Femininity; IS = Importance of Sex; D = Dominance; $\mathrm{T}=$ Toughness); ${ }^{\mathrm{e}}$ GRCS = Gender Role Conflict Scale (subscales: SPC = Success, Power, Competition; RE = Restrictive Emotionality; RABBM = Restrictive Affectionate Behavior Between Men; CBWFR $=$ Conflicts Between Work and Leisure - Family Relations); ${ }^{\mathrm{f}} \mathrm{FCV}-19 \mathrm{~S}=$ Fear of COVID-19 Scale; ${ }^{\mathrm{g}}$ CPSS $=$ COVID-19 Pandemic Stress Scale.

$*=p<.05$ 


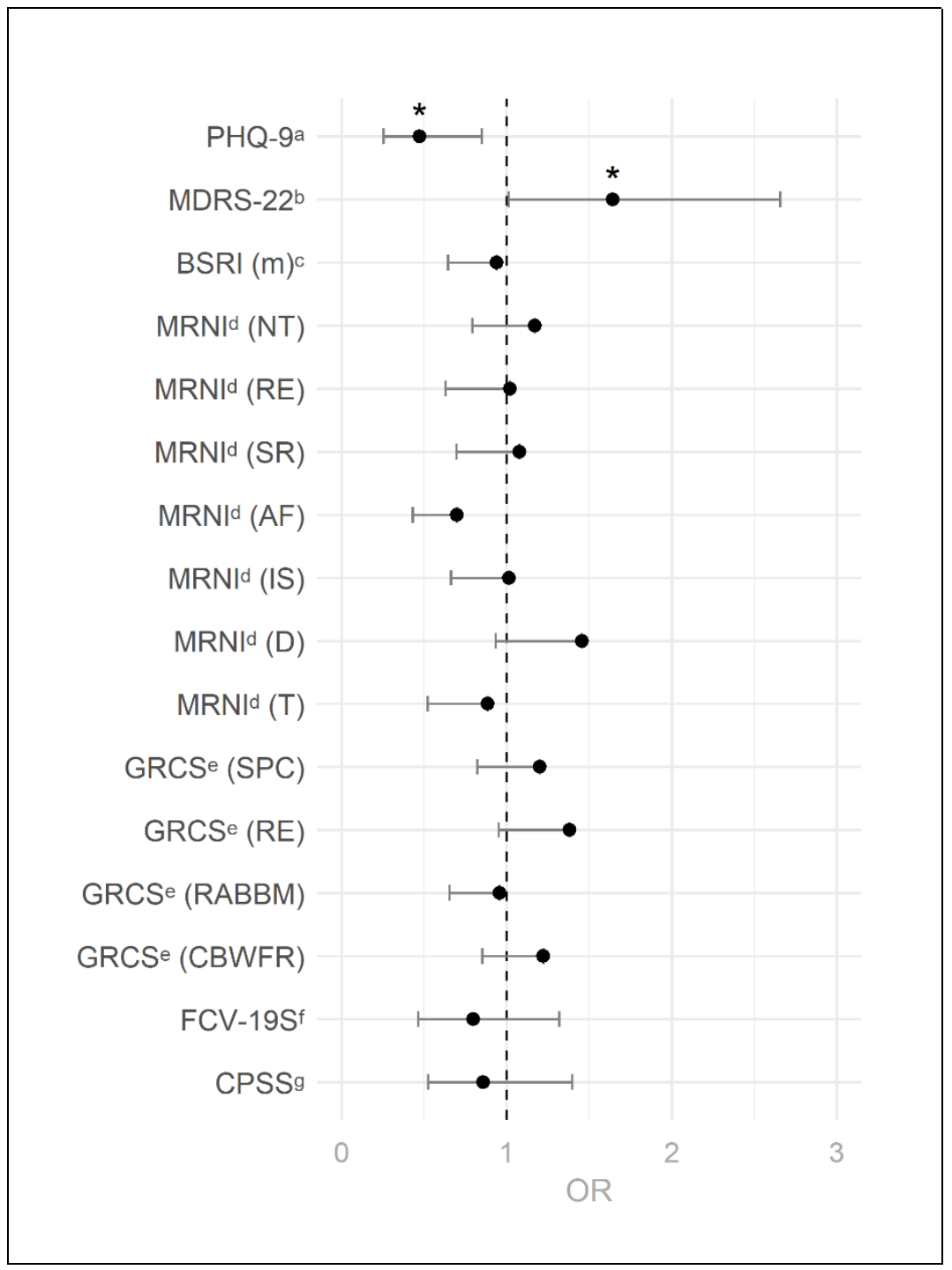

Figure 3. Standardized Odds Ratios for COVID-19 Infection and their two- and one-sided 95\% Confidence Intervals.

Note. $O R=$ Odds ratio

${ }^{\text {a }}$ PHQ-9 = Patient health Questionnaire - 9; ${ }^{\mathrm{b}}$ MDRS-22 = Male Depression Risk Scale -22 ; $^{\mathrm{c}}$ BSRI $(\mathrm{m})=$ Bem SexRole Inventory (masculinity subscale); ${ }^{\mathrm{d}} \mathrm{MRNI}=$ Male Role Norms Inventory (subscales: NT $=$ Negativity toward Sexual Minorities; $\mathrm{RE}=$ Restrictive Emotionality; $\mathrm{SR}=$ Selfreliance through Mechanical Skills; $\mathrm{AF}=$ Avoidance of Femininity; IS = Importance of Sex; $\mathrm{D}=$ Dominance; $\mathrm{T}=$ Toughness; ${ }^{\mathrm{e}}$ GRCS $=$ Gender Role Conflict Scale (subscales: $\mathrm{SPC}=$ Success, Power, Competition; RE $=$ Restrictive Emotionality; RABBM = Restrictive Affectionate Behavior Between Men; CBWFR = Conflicts Between Work and Leisure - Family Relations); ${ }^{\mathrm{f}} \mathrm{FCV}$-19S $=$ Fear of COVID-19 Scale; ${ }^{\mathrm{g}}$ CPSS $=$ COVID-19 Pandemic Stress Scale.

${ }^{*}=p<.05$ 


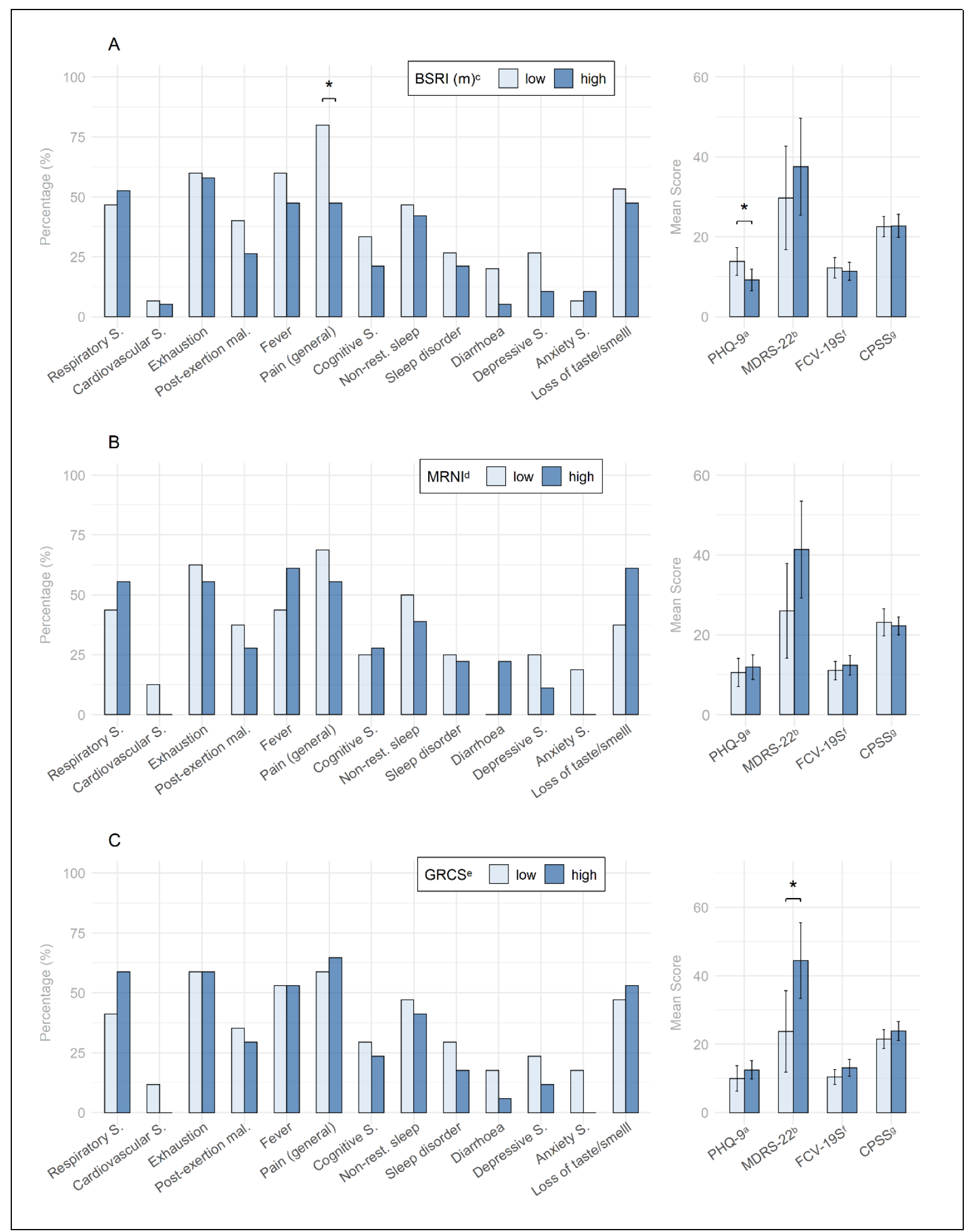

Figure 4. Differences in COVID-19 and Depression Symptoms, Fear of COVID-19, and COVID-19 Pandemic related Stress between men with low and high Traditional Masculinity. Note. $S .=$ symptoms, mal. $=$ malaise, low $/$ high $=$ below/above the median.

${ }^{\text {a }}$ PHQ-9 = Patient health Questionnaire - 9; ${ }^{b}$ MDRS-22 = Male Depression Risk Scale - 22; (masculinity subscale); ${ }^{\mathrm{d}} \mathrm{MRNI}=$ Male Role Norms Inventory; ${ }^{\mathrm{e}}$ GRCS $=$ Gender Role Conflict Scale; ${ }^{\mathrm{f}}$ FCV-19S $=$ Fear of COVID-19 Scale; ${ }^{\mathrm{g}}$ CPSS $=$ COVID-19 Pandemic Stress Scale. $^{*}=p<.05$ 


\section{Examining the role of traditional masculinity and depression in men's risk for contracting COVID-19}

Walther, A. ${ }^{1, *}$, Eggenberger, L. ${ }^{1}$, Grub, J. ${ }^{1}$, Ogrodniczuk, J.S. ${ }^{2}$, Seidler, Z.E. ${ }^{3,4}$, Rice, S.M. ${ }^{3,4}$, Kealy, D. ${ }^{2}$, Oliffe, J.L. ${ }^{5,6}$, Ehlert, U. ${ }^{1}$

${ }^{1}$ Clinical Psychology and Psychotherapy, University of Zurich, Zurich, Switzerland

${ }^{2}$ Department of Psychiatry, University of British Columbia, Vancouver, Canada

${ }^{3}$ Centre for Youth Mental Health, The University of Melbourne, Parkville, VIC, Australia

${ }^{4}$ Orygen, Melbourne, VIC, Australia

${ }^{5}$ School of Nursing, University of British Columbia, Vancouver, Canada

${ }^{6}$ Department of Nursing, University of Melbourne, Victoria, Australia

* Corresponding author

\section{Supplementary Material}

\section{Content}

S-Figure 1: Standardized Odds Ratios for COVID-19 Infection and their two- and one-sided 95\% Confidence Intervals

page 1

S-Table 1: Results of Mean Score Comparisons between Men with and without COVID-19 Infection

page 2

S-Table 2: Results of Binary Logistic Regression Analyses predicting COVID-19 Infection using Total Scores page 3

S-Table 3: Results of Binary Logistic Regression Analyses predicting COVID-19 Infection using Subscales

page 4

S-Discussion: Discussion part on traditional masculinity and specific COVID-19-related symptoms page 5 


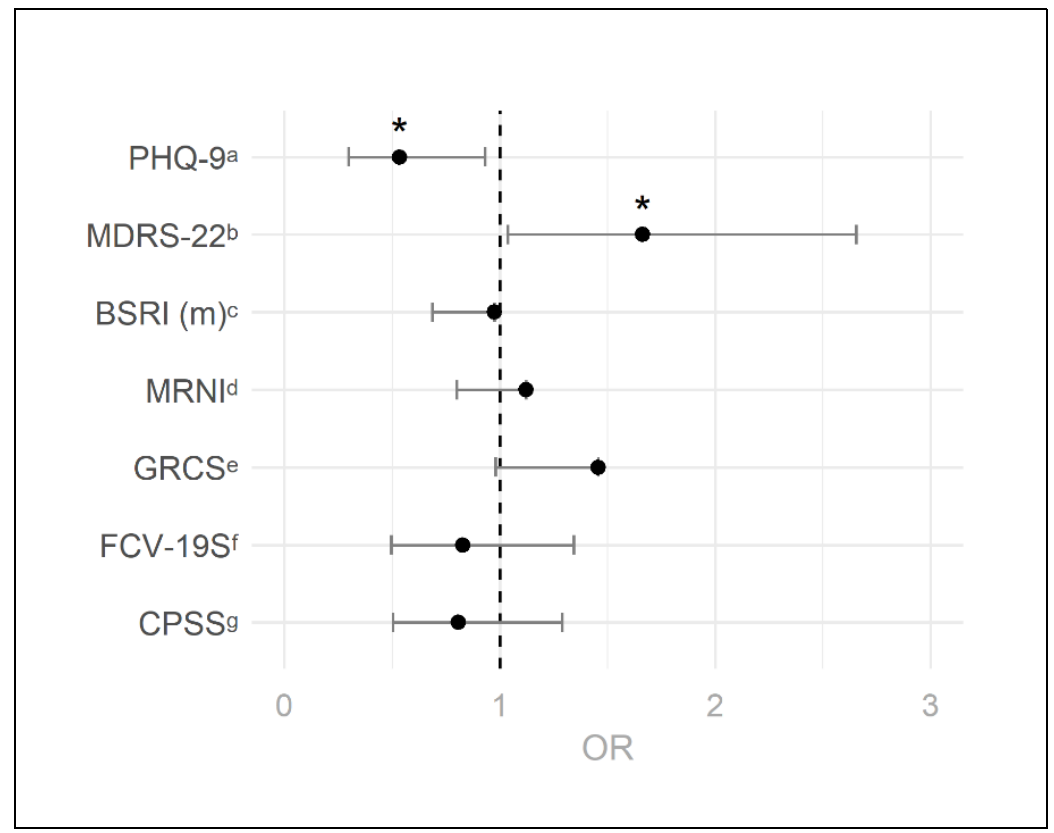

S-Figure 1. Standardized Odds Ratios for COVID-19 Infection and their two- and one-sided 95\% Confidence Intervals

Note. $O R=$ Odds ratio

${ }^{\mathrm{a}}$ PHQ-9 = Patient health Questionnaire - 9; ${ }^{\mathrm{b}}$ MDRS-22 = Male Depression Risk Scale $-22 ;{ }^{\mathrm{c}}$ BSRI $(\mathrm{m})=$ Bem SexRole Inventory (masculinity subscale) ${ }^{\mathrm{d}} \mathrm{MRNI}=$ Male Role Norms Inventory; ${ }^{\mathrm{e}} \mathrm{GRCS}=$ Gender Role Conflict Scale $;{ }^{\mathrm{f}}$ FCV-19S $=$ Fear of COVID-19 Scale; ${ }^{\mathrm{g}}$ CPSS $=$ COVID-19 Pandemic Stress Scale.

${ }^{*}=p<.05$ 
S-Table 1. Results of Mean Score Comparisons between Men with and without COVID-19 Infection

\begin{tabular}{|c|c|c|c|c|c|c|}
\hline \multirow{2}{*}{ Variable } & No CV19 & CV19 & \multirow{2}{*}{$t(d f)$} & \multirow{2}{*}{$p$} & \multirow{2}{*}{$p$ (corr.) } & \multirow{2}{*}{$d$} \\
\hline & $\mathrm{M}(S D)$ & $\mathrm{M}(S D)$ & & & & \\
\hline PHQ-9a & $12.7(6.6)$ & $11.3(6.3)$ & $1.21(488)$ & .225 & 1 & -.22 \\
\hline MDRS-22 ${ }^{b}$ & $28.8(20.3)$ & $34.1(24.4)$ & $-1.45(488)$ & .148 & 1 & .26 \\
\hline $\operatorname{BSRI}(\mathrm{m})^{\mathrm{c}}$ & $62.2(14.6)$ & $65.4(15.2)$ & $-1.20(488)$ & .116 & .668 & .21 \\
\hline MRNI $^{d}$ (tot.) & $44.9(20.7)$ & $54.4(28.9)$ & $-1.87(36)$ & $.035^{*}$ & .561 & .44 \\
\hline$(\mathrm{NT})$ & $4.3(3.1)$ & $5.6(4.8)$ & $-1.61(35)$ & .058 & .643 & .41 \\
\hline (RE) & $6.7(4.0)$ & $8.3(4.7)$ & $-2.12(488)$ & $.017^{*}$ & .308 & .38 \\
\hline (SR) & $10.5(4.9)$ & $11.6(5.4)$ & $-1.31(488)$ & .095 & .668 & .23 \\
\hline (AF) & $5.2(3.7)$ & $6.5(5.7)$ & $-1.27(35)$ & .106 & .668 & .33 \\
\hline (IS) & $4.7(3.1)$ & $5.7(3.9)$ & $-1.73(488)$ & $.042^{*}$ & .624 & .31 \\
\hline (D) & $4.4(2.9)$ & $6.1(5.0)$ & $-1.94(35)$ & $.030^{*}$ & .511 & .55 \\
\hline$(\mathrm{T})$ & $9.1(5.0)$ & $10.6(5.7)$ & $-1.66(488)$ & $.049^{*}$ & .624 & .30 \\
\hline GRCS $^{\mathrm{e}}$ (tot.) & 46.7 (12.2) & $51.7(16.0)$ & $-1.78(36)$ & $.042^{*}$ & .624 & .40 \\
\hline (SPC) & $13.1(4.8)$ & $14.9(6.0)$ & $-1.75(36)$ & $.044^{*}$ & .624 & .38 \\
\hline (RE) & $12.5(5.6)$ & $13.9(5.2)$ & $-1.43(488)$ & .076 & .667 & .26 \\
\hline (RABBM) & $7.7(3.7)$ & $8.7(5.0)$ & $-1.12(36)$ & .136 & .668 & .26 \\
\hline (CBWFR) & $13.4(5.2)$ & $14.1(6.3)$ & $-0.80(488)$ & .212 & .668 & .14 \\
\hline FCV-19S ${ }^{f}$ & $12.9(4.9)$ & $11.8(4.6)$ & $1.28(488)$ & .200 & 1 & -.23 \\
\hline CPSS $^{g}$ & $24.0(5.2)$ & $22.6(5.4)$ & $1.52(488)$ & .130 & 1 & -.27 \\
\hline
\end{tabular}

Note. One-sided $t$-tests were used for the BSRI (m), MRNI (including all its subscales), and GRCS (including all its subscales). $M=$ mean score, $S D=$ standard deviation, $t=t$-value, $d f=$ degrees of freedom, $p=p$-value, corr.$=$ corrected for multiple testing using the HolmBonferroni method, $d=$ Cohen's $d$.

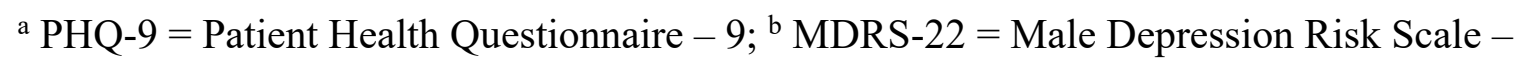
22; ${ }^{\mathrm{c}}$ BSRI $(\mathrm{m})=$ Bem Sex-Role Inventory (masculinity subscale); ${ }^{\mathrm{d}}$ MRNI = Male Role Norms Inventory (subscales: NT = Negativity toward Sexual Minorities; RE $=$ Restrictive Emotionality; SR = Self-reliance through Mechanical Skills; AF = Avoidance of Femininity; IS = Importance of Sex; D = Dominance; T = Toughness); ${ }^{\mathrm{e}}$ GRCS $=$ Gender Role Conflict Scale (subscales: SPC $=$ Success, Power, Competition; RE $=$ Restrictive Emotionality; RABBM = Restrictive Affectionate Behavior Between Men; CBWFR = Conflicts Between Work and Leisure - Family Relations); ${ }^{f}$ FCV-19S = Fear of COVID-19 Scale; ${ }^{\mathrm{g}}$ CPSS $=$ COVID-19 Pandemic Stress Scale.

${ }^{*}=p<.05$ 
S-Table 2. Results of Binary Logistic Regression Analyses predicting COVID-19 Infection using Total Scores

\begin{tabular}{|c|c|c|c|c|c|}
\hline Variable & $\beta^{1}(S E)^{1}$ & $\mathbf{O R}^{1}$ & $95 \%$ CI & $p$ & p (corr.) \\
\hline Intercept & $-2.94(0.23)$ & 0.05 & {$[0.03,0.08]$} & $<.001^{* * *}$ & $<.001 * * *$ \\
\hline PHQ-9a & $-0.63(0.29)$ & 0.53 & {$[0.30,0.93]$} & $.031^{*}$ & .338 \\
\hline MDRS-22 $^{b}$ & $0.51(0.24)$ & 1.66 & {$[1.04,2.65]$} & $.032^{*}$ & .338 \\
\hline $\operatorname{BSRI}(\mathrm{m})^{\mathrm{c}}$ & $-0.03(0.22)$ & 0.97 & {$[0.69]$,} & .395 & 1 \\
\hline MRNI $^{d}$ & $0.12(0.20)$ & 1.12 & {$[0.80, \underline{[}$} & .451 & 1 \\
\hline $\mathbf{G R C S}^{\mathrm{e}}$ & $0.38(0.24)$ & 1.46 & {$[0.98, \ldots[$} & .285 & .479 \\
\hline FCV-19S ${ }^{f}$ & $-0.19(0.25)$ & 0.83 & {$[0.49,1.34]$} & .460 & 1 \\
\hline CPSS $^{g}$ & $-0.21(0.24)$ & 0.81 & {$[0.50,1.29]$} & .369 & 1 \\
\hline \multicolumn{6}{|l|}{ Covariates } \\
\hline Age & $-0.21(0.21)$ & 0.81 & {$[0.51,1.18]$} & .318 & 1 \\
\hline Income & $-0.04(0.21)$ & 0.96 & {$[0.46,1.25]$} & .860 & 1 \\
\hline Education & $0.61(0.28)$ & 1.84 & {$[1.12,3.40]$} & $.031^{*}$ & .338 \\
\hline Sexual Orientation & $0.01(0.22)$ & 1.01 & {$[0.63,1.50]$} & .944 & 1 \\
\hline
\end{tabular}

\section{Goodness of Fit}

$$
\text { Information criteria } \quad \mathrm{AIC}=247.3 ; \quad \mathrm{BIC}=297.7 ; R^{2}=11.9 \%
$$

Likelihood-ratio test $\quad \chi^{2}=23.7 ; \quad d f=11 ; \quad p=.015 *$

Note. ${ }^{1}$ Coefficients $z$-standardized. One-sided $z$-tests were used for the BSRI (m), MRNI (including all its subscales), and GRCS (including all its subscales). $\beta=$ estimate, $S E=$ standard error, $C I=$ confidence interval, $p=p$-value, corr. $=$ corrected for multiple testing using the Holm-Bonferroni method, $R^{2}=$ Nagelkerke pseudo $R$-squared.

${ }^{\mathrm{a}}$ PHQ-9 = Patient Health Questionnaire - 9; ${ }^{\mathrm{b}}$ MDRS-22 = Male Depression Risk Scale $22 ;{ }^{c}$ BSRI $(\mathrm{m})=$ Bem Sex-Role Inventory (masculinity subscale); ${ }^{\mathrm{d}} \mathrm{MRNI}=$ Male Role Norms Inventory; ${ }^{\text {e }}$ GRCS $=$ Gender Role Conflict Scale; ${ }^{\mathrm{f}}$ FCV-19S $=$ Fear of COVID-19 Scale; ${ }^{\mathrm{g}}$ CPSS $=$ COVID-19 Pandemic Stress Scale.

${ }^{*}=p<.05,{ }^{* * *}=p<.001$ 
S-Table 3. Results of Binary Logistic Regression Analyses predicting COVID-19 Infection using Subscales

\begin{tabular}{|c|c|c|c|c|c|}
\hline Variable & $\beta^{1}(S E)^{1}$ & OR $^{1}$ & $95 \%$ CI & $p$ & $p$ (corr.) \\
\hline Intercept & $-2.99(0.24)$ & 0.05 & {$[0.03,0.08]$} & $<.001^{* * *}$ & $<.0011^{* * *}$ \\
\hline PHQ-9a & $-0.75(0.31)$ & 0.47 & {$[0.25,0.85]$} & $.015^{*}$ & .299 \\
\hline MDRS-22 ${ }^{b}$ & $0.50(0.24)$ & 1.64 & {$[1.01,2.66]$} & $.043^{*}$ & .766 \\
\hline BSRI (m) $)^{\mathfrak{c}}$ & $-0.06(0.23)$ & 0.94 & {$[0.64]$,} & .395 & 1 \\
\hline \multicolumn{6}{|l|}{ MRNI d subscales } \\
\hline$(\mathrm{NT})$ & $0.16(0.23)$ & 1.17 & {$[0.79$} & .245 & 1 \\
\hline (RE) & $0.02(0.29)$ & 1.02 & {$[0.63$,} & .471 & 1 \\
\hline (SR) & $0.07(0.26)$ & 1.08 & {$[0.69]$,} & .390 & 1 \\
\hline (AF) & $-0.36(0.29)$ & 0.70 & {$[0.43][$,} & .106 & 1 \\
\hline (IS) & $0.01(0.24)$ & 1.01 & {$[0.66$,} & .478 & 1 \\
\hline (D) & $0.38(0.27)$ & 1.46 & {$[0.93$} & .081 & 1 \\
\hline$(\mathrm{T})$ & $-0.12(0.32)$ & 0.88 & {$[0.52][$,} & .350 & 1 \\
\hline \multicolumn{6}{|l|}{ GRCS $^{\mathrm{e}}$ subscales } \\
\hline (SPC) & $0.18(0.23)$ & 1.20 & {$[0.82$,} & .214 & 1 \\
\hline (RE) & $0.32(0.23)$ & 1.38 & {$[0.95][$,} & .078 & 1 \\
\hline (RABBM) & $-0.04(0.23)$ & 0.96 & {$[0.65][$,} & .421 & 1 \\
\hline (CBWFR) & $0.20(0.22)$ & 1.22 & {$[0.85][$,} & .179 & 1 \\
\hline FCV-19S & $-0.23(0.26)$ & 0.80 & {$[0.46,1.32]$} & .393 & 1 \\
\hline CPSS $^{\mathrm{g}}$ & $-0.15(0.25)$ & 0.86 & {$[0.52,1.40]$} & .535 & 1 \\
\hline \multicolumn{6}{|l|}{ Covariates } \\
\hline Age & $-0.23(0.23)$ & 0.80 & {$[0.48,1.22]$} & .327 & 1 \\
\hline SES & $-0.04(0.18)$ & 0.96 & {$[0.49,1.23]$} & .819 & 1 \\
\hline Education & $0.62(0.29)$ & 1.86 & {$[1.11,3.51]$} & $.034^{*}$ & .647 \\
\hline Sexual Orientation & $-0.01(0.22)$ & 0.99 & {$[0.61,1.49]$} & .977 & 1 \\
\hline
\end{tabular}

\section{Goodness of Fit}

Information criteria $\quad \mathrm{AIC}=261.2 ; \quad \mathrm{BIC}=349.3 ; \quad R^{2}=13.9 \%$

Likelihood-ratio test $\quad \chi^{2}=27.8 ; \quad d f=20 ; \quad p=.114$

Note. ${ }^{1}$ Coefficients $z$-standardized. One-sided $z$-tests were used for the BSRI (m), MRNI (including all its subscales), and GRCS (including all its subscales). $\beta=$ estimate, $S E=$ standard error, $C I=$ confidence interval, $p=p$-value, corr . $=$ corrected for multiple testing using the Holm-Bonferroni method, $R^{2}=$ Nagelkerke pseudo $R$-squared.

${ }^{\text {a }}$ PHQ-9 = Patient Health Questionnaire - 9; ${ }^{\mathrm{b}}$ MDRS-22 = Male Depression Risk Scale 22; ${ }^{\mathrm{c}}$ BSRI $(\mathrm{m})=$ Bem Sex-Role Inventory (masculinity subscale); ${ }^{\mathrm{d}}$ MRNI = Male Role Norms Inventory (subscales: NT = Negativity toward Sexual Minorities; RE $=$ Restrictive Emotionality; SR = Self-reliance through Mechanical Skills; $\mathrm{AF}=$ Avoidance of Femininity; IS = Importance of Sex; $\mathrm{D}=$ Dominance; $\mathrm{T}=$ Toughness); ${ }^{\mathrm{e}} \mathrm{GRCS}=$ Gender Role Conflict Scale (subscales: SPC = Success, Power, Competition; RE = Restrictive Emotionality; RABBM = Restrictive Affectionate Behavior Between Men; CBWFR = Conflicts Between Work and Leisure - Family Relations); ${ }^{f}$ FCV-19S = Fear of COVID-19 Scale; ${ }^{\mathrm{g}}$ CPSS $=$ COVID-19 Pandemic Stress Scale.

${ }^{*}=p<.05$ 
S-Discussion: Discussion part on traditional masculinity and specific COVID-19-related symptoms

Possible group differences between men with high and low traditional masculinity regarding expression of COVID-19 symptoms in men who contracted COVID-19 were investigated. However, the exploratory analysis included only a subsample of 34 men reporting to have contracted COVID-19. As can be seen in Figure 4A, men with high traditional masculinity operationalized as high BSRI scores exhibited less general (i.e. pain symptoms, headache, joint pain, muscle pain, peripheral neuropathy) pain symptomatology (47.4\%) as compared to men with low traditional masculinity (80.0\%) and lower PHQ-9 scores as compared to men with low traditional masculinity. This is line with previous research showing studies using the BSRI-M to report higher masculine gender orientation to be positively associated with constructs such as self-esteem and self-confidence and negatively associated with depression and anxiety symptoms (Johnson \& McCoy, 2000; Long, 1986; O'Heron \& Orlofsky, 1990). Nevertheless, although non-significant due to the very small sample size, a clear picture is observable, that in general men with higher BSRI-M scores report overall less COVID-19 symptomatology. With regard to the MRNI-SF and the GRCS-SF this is not the case suggesting the masculinity measures based on the gender role strain paradigm in contrast to the gender role identity paradigm (BSRI-M) reveal more complex associations and suggest to examine specific subscales in order to identify, which specific role norms or gender role conflicts are related to symptom expression. 